\title{
Checklist das aves do Estado de São Paulo, Brasil
}

\author{
Luís Fábio Silveira ${ }^{1,2,4}$ \& Alexandre Uezu ${ }^{3}$ \\ ${ }^{1}$ Departamento de Zoologia, Universidade de São Paulo - USP, \\ CP 11461, CEP 05422-970, São Paulo, SP, Brasil \\ ${ }^{2}$ Curador associado das coleções ornitológicas, Museu de Zoologia, Universidade de São Paulo - USP, \\ CP 42494, CEP 04218-970, São Paulo, SP, Brasil \\ ${ }^{3}$ Instituto de Pesquisas Ecológicas, CP 47, CEP 19260-000, Nazaré Paulista, SP, Brasil \\ ${ }^{4}$ Autor para correspondência: Alexandre Uezu, e-mail: aleuezu@ipe.org.br
}

SILVEIRA, L.F. \& UEZU, A. Checklist of birds from São Paulo State, Brazil. Biota Neotrop. 11(1a): http:// www.biotaneotropica.org.br/v11n1a/en/abstract?inventory+bn0061101a2011.

\begin{abstract}
Species lists are essential to understand both temporal and distributional patterns of taxa. Based on data compiled by CEO (Centro de Estudos Ornitológicos), Willis and Oniki (2003), and from a search of more than 50 theses, dissertations, monographs and technical works, we listed all bird species recorded in the State of São Paulo. These records are composed of skins and other evidence collected and deposited in collections, and on photographs and voice samples. A total of 793 species were registered, distributed in 25 orders and 85 families, and corresponding to $45 \%$ of the Brazilian avifauna. Reasons for this high diversity are related to the environmental diversity found in the state, influenced by altitudinal and geographical ranges, different phytophysiognomies, presence of a coastal region, and areas of contact between forest ecosystems and Cerrado. Results of the Biota project contributed to a better understanding of how birds respond to anthropogenic alterations of the environment, such as habitat fragmentation. The main ornithological research groups are still based in universities and museums. Deficiencies of knowledge concerning bird studies in São Paulo are related to the lack of standardization of survey methodologies; paucity in the monitoring of threatened species in the long term; restricted knowledge about species capacity to use matrix; and lack of refinement in the delimitation of evolutionary units and their distribution, which is essential for species reintroduction in regions where they have gone extinct.
\end{abstract}

Keywords: birds, biodiversity of the State of São Paulo, BIOTA/FAPESP Program.

Number of species: In the world: 11,000, in Brazil: 1,825, estimated in São Paulo State: 793.

SILVEIRA, L.F. \& UEZU, A. Checklist das aves do Estado de São Paulo, Brasil. Biota Neotrop. 11(1a): http:// www.biotaneotropica.org.br/v11n1a/pt/abstract?inventory+bn0061101a2011.

Resumo: As listas de espécies são fundamentais para entender os padrões de distribuição espaciais e temporais dos táxons. A partir das bases de dados compiladas pelo CEO - Centro de Estudos Ornitológicos, do levantamento de Willis \& Oniki (2003) e de buscas feitas em mais de 50 teses e dissertações, além de monografias e trabalhos técnicos, foram listadas todas as aves já registradas no Estado de São Paulo. Esses registros são compostos por peles ou outros materiais coletados e depositados em coleções, além de fotografias ou gravações. No total, foram registradas 793 espécies, distribuídas em 25 ordens e 85 famílias, correspondendo a cerca de $45 \%$ da avifauna brasileira. Um dos principais motivos para essa alta diversidade é a diversidade de ambientes encontrados no Estado, influenciados pelo gradiente altitudinal e geográfico, as diferentes fitofisionomias, a presença da região costeira, e o contato entre áreas florestais e Cerrados. O projeto Biota contribuiu principalmente no entendimento de como esse grupo responde às mudanças antrópicas do ambiente, como a fragmentação do habitat. Os principais grupos de pesquisa em ornitologia ainda estão alocados nas universidades e nos museus. As principais lacunas do conhecimento sobre as aves estão relacionadas à falta de padronização das metodologias para se inventariar este grupo, aos espaços geográficos sem amostragens, à falta de monitoramento em longo prazo das espécies ameaçadas de extinção, ao conhecimento limitado da capacidade das espécies em usarem as matrizes do entorno e ao pouco refinamento das delimitações das unidades evolutivas, que podem ser usadas na reintrodução das espécies em áreas em que estas não mais ocorrem.

Palavras-chave: aves, biota paulista, Programa BIOTA/FAPESP.

Número de espécies: No mundo: 11.000, no Brasil: 1.825, estimadas no estado de São Paulo: 793. 


\section{Introdução}

O táxon Aves é composto por aproximadamente 11.000 espécies, das quais 1.825 foram registradas no Brasil (Comitê Brasileiro de Registros Ornitológicos 2009). Existem ainda mais de 20.000 subespécies, o que indica que o número de unidades evolutivas válidas pode estar grandemente subestimado (Silveira \& Olmos 2007). As aves formam o grupo de vertebrados mais bem conhecido sob qualquer aspecto quando comparados com outros grupos de vertebrados. A diversidade, os hábitos e o comportamento das espécies fazem com que esse grupo seja habitualmente utilizado em monitoramentos de impactos ambientais, já que respondem rapidamente às alterações no seu ambiente (Uezu et al. 2005).

A elaboração de uma lista de espécies com base em determinados critérios é o primeiro passo para se compreender a distribuição das espécies e, em monitoramentos periódicos, verificar as tendências populacionais dos táxons listados. Uma lista de espécies é ainda uma ferramente indispensável para a elaboração de políticas de conservação em diversos níveis. Listas de caráter nacional têm sido elaboradas (e.g. Comitê Brasileiro de Registros Ornitológicos 2009) e servem como uma referência para se avaliar o grau de conhecimento de um determinado grupo e para apontar possíveis lacunas de conhecimento, incentivando, por exemplo, a documentação de determinados táxons conhecidos em uma região apenas por relatos. Dessa forma, os objetivos deste trabalho é apresentar a lista das espécies de Aves já registradas no Estado de São Paulo.

\section{Metodologia}

A avifauna do Estado de São Paulo pode ser considerada como bastante bem conhecida, e os seus primeiros inventários datam ainda do final do século XIX. A presente listagem teve como base duas indispensáveis compilações sobre a avifauna do estado. O Centro de Estudos Ornitológicos (CEO) vem organizando e atualizando a lista das aves paulistas, incluindo a data do primeiro registro e a forma de documentação para cada táxon. Willis \& Oniki (2003) publicaram a lista das aves do estado, incluindo dados sobre a sua distribuição. Além disso, a listagem publicada por Silveira et al. (2009) foi revista e atualizada. A presente listagem apresenta as espécies de aves que contém registros documentados, sejam eles através de peles ou outro material coletado e depositado em uma coleção, fotografia ou gravação, seguindo basicamente os mesmos critérios apresentados em Silveira et al. (2009). Dessa forma, algumas espécies constantes na listagem do CEO (Figueiredo 2002) não foram consideradas no presente trabalho, como Pyrrhura leucotis (Kuhl, 1820) e Paroaria dominicana (Linnaeus, 1758), sendo a primeira um erro de identificação e, a segunda, uma espécie endêmica da Caatinga e que eventualmente pode ser encontrada em liberdade na cidade de São Paulo e outros centros urbanos, como resultado de escapes de cativeiro.

Silveira et al. (2009) listam algumas espécies que possuíam alguma citação para o estado de São Paulo, mas que não contavam com registros documentados (Apêndice 1). Desde a publicação dessa lista apenas uma espécie, Pseudocolopteryx sclateri (Oustalet, 1892), foi incluída na lista primária. Em maio de 2010 essa ave foi fotografada em um brejo no município de Itaquaquecetuba por Guilherme Serpa, Wagner Nogueira, Emerson Kaseker e Helberth Cardoso. Além disso, Dendroica cerulea (Wilson, 1810), foi transferida para a lista das espécies de ocorrência duvidosa no Estado (Apêndice 1), aguardando uma melhor documentação para retornar à lista principal. A presente lista foi atualizada com os registros divulgados até julho de 2010, entre esses estudos constam mais de 50 teses e dissertações, além de monografias e trabalhos técnicos (como planos de manejo de Unidades de Conservação) realizadas no estado. Em muitos desses estudos utilizou-se a metodologia de rede de neblina, o que garante uma maior certeza na identificação das espécies. Encoraja-se a publicação de registros documentados (preferencialmente através de exemplares) das espécies constantes no Apêndice.

\section{Resultados e Discussão}

Foram registradas 793 espécies no estado de São Paulo, que representam 25 ordens e 85 famílias (Tabela 1). Esse número é ligeiramente superior ao apresentado por Silveira et al. (2009) e corresponde a aproximadamente $45 \%$ da avifauna brasileira, que conta com 1.825 espécies (Comitê Brasileiro de Registros Ornitológicos 2009). Mais de $95 \%$ dos táxons possui sua presença em São Paulo documentada através de espécimes-testemunho depositados em coleções e/ou foi repetidamente observada de forma independente no estado, o que permite inferências razoavelmente seguras sobre o seu status de residente ou migratória, além de possibilitar análises mais refinadas sobre as suas tendências populacionais ao longo do tempo (veja Silveira et al. 2009).

Embora não seja um dos Estados com maior extensão territorial no Brasil, São Paulo conta com uma grande diversidade de ambientes dentro dos biomas Costeiros, Mata Atlântica e Cerrado, que propiciam hábitats para um grande número de espécies de aves. Entre as florestas podemos citar a presença da Floresta Ombrófila densa, Floresta Ombrófila mista e a Floresta Estacional Semidecidual. Na zona costeira ainda destacamse os maguezais e a Restinga. E a presença de uma costa com praias e outros ambientes associados ao mar atrai aves migratórias do Hemisfério Norte e do sul da América do Sul, como maçaricos e andorinhas-do-mar. A Mata Atlântica, tanto na Serras do Mar e da Mantiqueira quanto no maciço de Paranapiacaba apresentam um expressivo gradiente altitudinal, variando do nível do mar até cerca de $2.800 \mathrm{~m}$, permitindo a sua colonização por espécies que utilizam faixas de altitude específicas. As matas semideciduais do interior permitem que muitas espécies da Mata Atlântica e do Cerrado entrem em contato. O Cerrado paulista, com as suas mais diversas fitofisionomias, abriga uma porção significativa das espécies já registradas nesse bioma e também proporciona hábitat para dezenas de espécies migratórias, como aquelas do gênero Sporophila. As matas de galeria, de maneira similar ao observado nas matas semideciduais, possibilitam que espécies florestais do Cerrado entrem em contato com aquelas da Mata Atlântica.

\section{Principais Avanços Relacionados ao Programa BIOTA/FAPESP}

Os principais avanços do Programa BIOTA para o grupo de aves estão relacionados ao aumento de conhecimento de como as espécies respondem às alterações antrópicas do ambiente. Entre os temas que foram aprofundados temos: perda e fragmentação do habitat (Develey \& Metzger 2005, Martensen et al. 2008), o efeito da presença de corredores conectando fragmentos (Uezu et al. 2005), o efeito do histórico de perturbação na diversidade atual (Metzger et al. 2009), a capacidade das espécies em se deslocarem pela matriz (Boscolo et al. 2008) e o efeito da caça (Bernardo et al. no prelo). Essas informações são essenciais para propor formas de manejo mais adequadas a fim de diminuir as pressões sobre esse grupo e conservar a diversidade ainda presente. Esses conhecimentos são especialmente úteis na indicação de áreas prioritárias para conservação (e.g. proposição de unidades de conservação), seleção de espécies indicadoras, criação de corredores ecológicos (em escalas locais e geográficas) e seleção de áreas para restauração (Silva et al. 2008).

\section{Principais Grupos de Pesquisa}

Os principais grupos de pesquisa em ornitologia no estado de São Paulo estão concentrados em diversos departamentos (e.g. Zoologia, Ecologia, Biologia, Genética) nas universidades públicas estaduais 
Tabela 1. Lista das espécies de Aves do Estado de São Paulo. A sistemática e a taxonomia seguem Comitê Brasileiro de Registros Ornitológicos (2009).

Table 1. List of bird species from State of São Paulo. The systematic and taxonomy is in accordance with Comitê Brasileiro de Registros Ornitológicos (2009).

\begin{tabular}{|c|c|}
\hline Nome do táxon & Nome em português \\
\hline \multicolumn{2}{|l|}{ Struthioniformes Latham, 1790} \\
\hline \multicolumn{2}{|l|}{ Rheidae Bonaparte, 1849} \\
\hline Rhea americana (Linnaeus, 1758) & ema \\
\hline \multicolumn{2}{|l|}{ Tinamiformes Huxley, 1872} \\
\hline \multicolumn{2}{|l|}{ Tinamidae Gray, 1840} \\
\hline Tinamus solitarius (Vieillot, 1819) & macuco \\
\hline Crypturellus obsoletus (Temminck, 1815) & inhambuguaçu \\
\hline Crypturellus undulatus (Temminck, 1815) & jaó \\
\hline Crypturellus noctivagus (Wied, 1820) & jaó-do-sul \\
\hline Crypturellus parvirostris (Wagler, 1827) & inhambu-chororó \\
\hline Crypturellus tataupa (Temminck, 1815) & inhambu-chintã \\
\hline Rhynchotus rufescens (Temminck, 1815) & perdiz \\
\hline Nothura minor (Spix, 1825) & codorna-mineira \\
\hline Nothura maculosa (Temminck, 1815) & codorna-amarela \\
\hline Taoniscus nanus (Temminck, 1815) & inhambu-carapé \\
\hline \multicolumn{2}{|l|}{ Anseriformes Linnaeus, 1758} \\
\hline \multicolumn{2}{|l|}{ Anhimidae Stejneger, 1885} \\
\hline Anhima cornuta (Linnaeus, 1766) & anhuma \\
\hline Chauna torquata (Oken, 1816) & tachã \\
\hline \multicolumn{2}{|l|}{ Anatidae Leach, 1820} \\
\hline Dendrocygna bicolor (Vieillot, 1816) & marreca-caneleira \\
\hline Dendrocygna viduata (Linnaeus, 1766) & irerê \\
\hline Dendrocygna autumnalis (Linnaeus, 1758) & asa-branca \\
\hline Cygnus melancoryphus (Molina, 1782) & cisne-de-pescoço-preto \\
\hline Neochen jubata (Spix, 1825) & pato-corredor \\
\hline Cairina moschata (Linnaeus, 1758) & pato-do-mato \\
\hline Sarkidiornis sylvicola Ihering \& Ihering, 1907 & pato-de-crista \\
\hline Callonetta leucophrys (Vieillot, 1816) & marreca-de-coleira \\
\hline Amazonetta brasiliensis (Gmelin, 1789) & pé-vermelho \\
\hline Anas flavirostris Vieillot, 1816 & marreca-pardinha \\
\hline Anas georgica Gmelin, 1789 & marreca-parda \\
\hline Anas bahamensis Linnaeus, 1758 & marreca-toicinho \\
\hline Anas versicolor Vieillot, 1816 & marreca-cricri \\
\hline Anas discors Linnaeus, 1766 & marreca-de-asa-azul \\
\hline Netta erythrophthalma (Wied, 1832) & paturi-preta \\
\hline Netta peposaca (Vieillot, 1816) & marrecão \\
\hline Mergus octosetaceus Vieillot, 1817 & pato-mergulhão \\
\hline Nomonyx dominica (Linnaeus, 1766) & marreca-de-bico-roxo \\
\hline \multicolumn{2}{|l|}{ Galliformes Linnaeus, 1758} \\
\hline \multicolumn{2}{|l|}{ Cracidae Rafinesque, 1815} \\
\hline Ortalis guttata (Spix, 1825) & aracuã \\
\hline Penelope superciliaris Temminck, 1815 & jacupemba \\
\hline Penelope obscura Temminck, 1815 & jacuaçu \\
\hline Aburria jacutinga (Spix, 1825) & jacutinga \\
\hline Crax fasciolata Spix, 1825 & mutum-de-penacho \\
\hline \multicolumn{2}{|l|}{ Odontophoridae Gould, 1844} \\
\hline Odontophorus capueira (Spix, 1825) & uru \\
\hline \multicolumn{2}{|l|}{ Podicipediformes Fürbringer, 1888} \\
\hline \multicolumn{2}{|l|}{ Podicipedidae Bonaparte, 1831} \\
\hline Rollandia rolland (Quoy \& Gaimard, 1824) & mergulhão-de-orelha-branca \\
\hline
\end{tabular}


Tabela 1. Continuação...

\begin{tabular}{|c|c|}
\hline Nome do táxon & Nome em português \\
\hline Tachybaptus dominicus (Linnaeus, 1766) & mergulhão-pequeno \\
\hline Podilymbus podiceps (Linnaeus, 1758) & mergulhão-caçador \\
\hline Podicephorus major (Boddaert, 1783) & mergulhão-grande \\
\hline Podiceps occipitalis Garnot, 1826 & mergulhão-de-orelha-amarela \\
\hline \multicolumn{2}{|l|}{ Sphenisciformes Sharpe, 1891} \\
\hline \multicolumn{2}{|l|}{ Spheniscidae Bonaparte, 1831} \\
\hline Spheniscus magellanicus (Forster, 1781) & pingüim-de-magalhães \\
\hline \multicolumn{2}{|l|}{ Procellariiformes Fürbringer, 1888} \\
\hline \multicolumn{2}{|l|}{ Diomedeidae Gray, 1840} \\
\hline Phoebetria fusca (Hilsenberg, 1822) & piau-preto \\
\hline Thalassarche chlororhynchos (Gmelin, 1789) & albatroz-de-nariz-amarelo \\
\hline Thalassarche melanophris (Temminck, 1828) & albatroz-de-sobrancelha \\
\hline Thalassarche chrysostoma (Forster, 1785) & albatroz-de-cabeça-cinza \\
\hline Diomedea exulans Linnaeus, 1758 & albatroz-gigante \\
\hline Diomedea dabbenena Mathews, 1929 & albatroz-de-tristão \\
\hline \multicolumn{2}{|l|}{ Procellariidae Leach, 1820} \\
\hline Macronectes giganteus (Gmelin, 1789) & petrel-gigante \\
\hline Macronectes halli Mathews, 1912 & petrel-gigante-do-norte \\
\hline Fulmarus glacialoides (Smith, 1840) & pardelão-prateado \\
\hline Daption capense (Linnaeus, 1758) & pomba-do-cabo \\
\hline Pterodroma incerta (Schlegel, 1863) & grazina-de-barriga-branca \\
\hline Pachyptila desolata (Gmelin, 1789) & faigão-rola \\
\hline Pachyptila belcheri (Mathews, 1912) & faigão-de-bico-fino \\
\hline Procellaria aequinoctialis Linnaeus, 1758 & pardela-preta \\
\hline Procellaria conspicillata Gould, 1844 & pardela-de-óculos \\
\hline Calonectris borealis (Cory, 1881) & bobo-grande \\
\hline Puffinus griseus (Gmelin, 1789) & bobo-escuro \\
\hline Puffinus gravis (O’Reilly, 1818) & bobo-grande-de-sobre-branco \\
\hline Puffinus puffinus (Brünnich, 1764) & bobo-pequeno \\
\hline \multicolumn{2}{|l|}{ Hydrobatidae Mathews, 1912} \\
\hline Fregetta grallaria (Vieillot, 1818) & painho-de-barriga-branca \\
\hline Oceanites oceanicus (Kuhl, 1820) & alma-de-mestre \\
\hline \multicolumn{2}{|l|}{ Pelecaniformes Sharpe, 1891} \\
\hline \multicolumn{2}{|l|}{ Sulidae Reichenbach, 1849} \\
\hline Sula dactylatra Lesson, 1831 & atobá-grande \\
\hline Sula leucogaster (Boddaert, 1783) & atobá-pardo \\
\hline \multicolumn{2}{|l|}{ Phalacrocoracidae Reichenbach, 1849} \\
\hline Phalacrocorax brasilianus (Gmelin, 1789) & biguá \\
\hline \multicolumn{2}{|l|}{ Anhingidae Reichenbach, 1849} \\
\hline Anhinga anhinga (Linnaeus, 1766) & biguatinga \\
\hline \multicolumn{2}{|l|}{ Fregatidae Degland \& Gerbe, 1867} \\
\hline Fregata magnificens Mathews, 1914 & tesourão \\
\hline \multicolumn{2}{|l|}{ Ciconiiformes Bonaparte, 1854} \\
\hline \multicolumn{2}{|l|}{ Ardeidae Leach, 1820} \\
\hline Tigrisoma lineatum (Boddaert, 1783) & socó-boi \\
\hline Tigrisoma fasciatum (Such, 1825) & socó-boi-escuro \\
\hline Cochlearius cochlearius (Linnaeus, 1766) & arapapá \\
\hline Botaurus pinnatus (Wagler, 1829) & socó-boi-baio \\
\hline Ixobrychus exilis (Gmelin, 1789) & socoí-vermelho \\
\hline Ixobrychus involucris (Vieillot, 1823) & socoí-amarelo \\
\hline Nycticorax nycticorax (Linnaeus, 1758) & savacu \\
\hline
\end{tabular}


Tabela 1. Continuação...

\begin{tabular}{|c|c|}
\hline Nome do táxon & Nome em português \\
\hline Nyctanassa violacea (Linnaeus, 1758) & savacu-de-coroa \\
\hline Butorides striata (Linnaeus, 1758) & socozinho \\
\hline Bubulcus ibis (Linnaeus, 1758) & garça-vaqueira \\
\hline Ardea cocoi Linnaeus, 1766 & garça-moura \\
\hline Ardea alba Linnaeus, 1758 & garça-branca-grande \\
\hline Syrigma sibilatrix (Temminck, 1824) & maria-faceira \\
\hline Pilherodius pileatus (Boddaert, 1783) & garça-real \\
\hline Egretta thula (Molina, 1782) & garça-branca-pequena \\
\hline Egretta caerulea (Linnaeus, 1758) & garça-azul \\
\hline \multicolumn{2}{|l|}{ Threskiornithidae Poche, 1904} \\
\hline Eudocimus ruber (Linnaeus, 1758) & guará \\
\hline Plegadis chihi (Vieillot, 1817) & caraúna-de-cara-branca \\
\hline Mesembrinibis cayennensis (Gmelin, 1789) & coró-coró \\
\hline Phimosus infuscatus (Lichtenstein, 1823) & tapicuru-de-cara-pelada \\
\hline Theristicus caudatus (Boddaert, 1783) & curicaca \\
\hline Platalea ajaja Linnaeus, 1758 & colhereiro \\
\hline \multicolumn{2}{|l|}{ Ciconiidae Sundevall, 1836} \\
\hline Ciconia maguari (Gmelin, 1789) & maguari \\
\hline Jabiru mycteria (Lichtenstein, 1819) & tuiuiú \\
\hline Mycteria americana Linnaeus, 1758 & cabeça-seca \\
\hline \multicolumn{2}{|l|}{ Phoenicopteriformes Fürbringer, 1888} \\
\hline \multicolumn{2}{|l|}{ Phoenicopteridae Bonaparte, 1831} \\
\hline Phoenicopterus chilensis Molina, 1782 & flamingo-chileno \\
\hline \multicolumn{2}{|l|}{ Cathartiformes Seebohm, 1890} \\
\hline \multicolumn{2}{|l|}{ Cathartidae Lafresnaye, 1839} \\
\hline Cathartes aura (Linnaeus, 1758) & urubu-de-cabeça-vermelha \\
\hline Cathartes burrovianus Cassin, 1845 & urubu-de-cabeça-amarela \\
\hline Coragyps atratus (Bechstein, 1793) & urubu-de-cabeça-preta \\
\hline Sarcoramphus papa (Linnaeus, 1758) & urubu-rei \\
\hline \multicolumn{2}{|l|}{ Falconiformes Bonaparte, 1831} \\
\hline \multicolumn{2}{|l|}{ Pandionidae Bonaparte, 1854} \\
\hline Pandion haliaetus (Linnaeus, 1758) & águia-pescadora \\
\hline \multicolumn{2}{|l|}{ Accipitridae Vigors, 1824} \\
\hline Leptodon cayanensis (Latham, 1790) & gavião-de-cabeça-cinza \\
\hline Chondrohierax uncinatus (Temminck, 1822) & caracoleiro \\
\hline Elanoides forficatus (Linnaeus, 1758) & gavião-tesoura \\
\hline Gampsonyx swainsonii Vigors, 1825 & gaviãozinho \\
\hline Elanus leucurus (Vieillot, 1818) & gavião-peneira \\
\hline Rostrhamus sociabilis (Vieillot, 1817) & gavião-caramujeiro \\
\hline Harpagus diodon (Temminck, 1823) & gavião-bombachinha \\
\hline Ictinia plumbea (Gmelin, 1788) & sovi \\
\hline Circus cinereus Vieillot, 1816 & gavião-cinza \\
\hline Circus buffoni (Gmelin, 1788) & gavião-do-banhado \\
\hline Accipiter poliogaster (Temminck, 1824) & tauató-pintado \\
\hline Accipiter superciliosus (Linnaeus, 1766) & gavião-miudinho \\
\hline Accipiter striatus Vieillot, 1808 & gavião-miúdo \\
\hline Accipiter bicolor (Vieillot, 1817) & gavião-bombachinha-grande \\
\hline Geranospiza caerulescens (Vieillot, 1817) & gavião-pernilongo \\
\hline Leucopternis lacernulatus (Temminck, 1827) & gavião-pombo-pequeno \\
\hline Leucopternis polionotus (Kaup, 1847) & gavião-pombo-grande \\
\hline Buteogallus aequinoctialis (Gmelin, 1788) & caranguejeiro \\
\hline
\end{tabular}


Tabela 1. Continuação...

\begin{tabular}{|c|c|}
\hline Nome do táxon & Nome em português \\
\hline Buteogallus urubitinga (Gmelin, 1788) & gavião-preto \\
\hline Heterospizias meridionalis (Latham, 1790) & gavião-caboclo \\
\hline Harpyhaliaetus coronatus (Vieillot, 1817) & águia-cinzenta \\
\hline Busarellus nigricollis (Latham, 1790) & gavião-belo \\
\hline Parabuteo unicinctus (Temminck, 1824) & gavião-asa-de-telha \\
\hline Percnohierax leucorrhous (Quoy \& Gaimard, 1824) & gavião-de-sobre-branco \\
\hline Rupornis magnirostris (Gmelin, 1788) & gavião-carijó \\
\hline Buteo albicaudatus Vieillot, 1816 & gavião-de-rabo-branco \\
\hline Buteo nitidus (Latham, 1790) & gavião-pedrês \\
\hline Buteo swainsoni Bonaparte, 1838 & gavião-papa-gafanhoto \\
\hline Buteo brachyurus Vieillot, 1816 & gavião-de-cauda-curta \\
\hline Buteo albonotatus Kaup, 1847 & gavião-de-rabo-barrado \\
\hline Morphnus guianensis (Daudin, 1800) & uiraçu-falso \\
\hline Harpia harpyja (Linnaeus, 1758) & gavião-real \\
\hline Spizaetus tyrannus (Wied, 1820) & gavião-pega-macaco \\
\hline Spizaetus melanoleucus (Vieillot, 1816) & gavião-pato \\
\hline Spizaetus ornatus (Daudin, 1800) & gavião-de-penacho \\
\hline \multicolumn{2}{|l|}{ Falconidae Leach, 1820} \\
\hline Ibycter americanus (Boddaert, 1783) & gralhão \\
\hline Caracara plancus (Miller, 1777) & caracará \\
\hline Milvago chimachima (Vieillot, 1816) & carrapateiro \\
\hline Milvago chimango (Vieillot, 1816) & chimango \\
\hline Herpetotheres cachinnans (Linnaeus, 1758) & acauã \\
\hline Micrastur ruficollis (Vieillot, 1817) & falcão-caburé \\
\hline Micrastur semitorquatus (Vieillot, 1817) & falcão-relógio \\
\hline Falco sparverius Linnaeus, 1758 & quiriquiri \\
\hline Falco rufigularis Daudin, 1800 & cauré \\
\hline Falco deiroleucus Temminck, 1825 & falcão-de-peito-laranja \\
\hline Falco femoralis Temminck, 1822 & falcão-de-coleira \\
\hline Falco peregrinus Tunstall, 1771 & falcão-peregrino \\
\hline \multicolumn{2}{|l|}{ Gruiformes Bonaparte, 1854} \\
\hline \multicolumn{2}{|l|}{ Aramidae Bonaparte, 1852} \\
\hline Aramus guarauna (Linnaeus, 1766) & carão \\
\hline \multicolumn{2}{|l|}{ Rallidae Rafinesque, 1815} \\
\hline Coturnicops notatus (Gould, 1841) & pinto-d'água-carijó \\
\hline Micropygia schomburgkii (Schomburgk, 1848) & maxalalagá \\
\hline Rallus longirostris Boddaert, 1783 & saracura-matraca \\
\hline Aramides mangle (Spix, 1825) & saracura-do-mangue \\
\hline Aramides cajanea (Statius Muller, 1776) & saracura-três-potes \\
\hline Aramides saracura (Spix, 1825) & saracura-do-mato \\
\hline Amaurolimnas concolor (Gosse, 1847) & saracura-lisa \\
\hline Laterallus viridis (Statius Muller, 1776) & sanã-castanha \\
\hline Laterallus melanophaius (Vieillot, 1819) & sanã-parda \\
\hline Laterallus exilis (Temminck, 1831) & sanã-do-capim \\
\hline Laterallus leucopyrrhus (Vieillot, 1819) & sanã-vermelha \\
\hline Laterallus xenopterus Conover, 1934 & sanã-de-cara-ruiva \\
\hline Porzana flaviventer (Boddaert, 1783) & sanã-amarela \\
\hline Porzana albicollis (Vieillot, 1819) & sanã-carijó \\
\hline Neocrex erythrops (Sclater, 1867) & turu-turu \\
\hline Pardirallus maculatus (Boddaert, 1783) & saracura-carijó \\
\hline Pardirallus nigricans (Vieillot, 1819) & saracura-sanã \\
\hline
\end{tabular}


Tabela 1. Continuação...

\begin{tabular}{|c|c|}
\hline Nome do táxon & Nome em português \\
\hline Pardirallus sanguinolentus (Swainson, 1837) & saracura-do-banhado \\
\hline Gallinula chloropus (Linnaeus, 1758) & frango-d'água-comum \\
\hline Gallinula melanops (Vieillot, 1819) & frango-d'água-carijó \\
\hline Porphyrio martinica (Linnaeus, 1766) & frango-d'água-azul \\
\hline Porphyrio flavirostris (Gmelin, 1789) & frango-d'água-pequeno \\
\hline Fulica armillata Vieillot, 1817 & carqueja-de-bico-manchado \\
\hline Fulica rufifrons Philippi \& Landbeck, 1861 & carqueja-de-escudo-vermelho \\
\hline Fulica leucoptera Vieillot, 1817 & carqueja-de-bico-amarelo \\
\hline \multicolumn{2}{|l|}{ Heliornithidae Gray, 1840} \\
\hline Heliornis fulica (Boddaert, 1783) & picaparra \\
\hline \multicolumn{2}{|l|}{ Cariamidae Bonaparte, 1850} \\
\hline Cariama cristata (Linnaeus, 1766) & seriema \\
\hline \multicolumn{2}{|l|}{ Charadriiformes Huxley, 1867} \\
\hline \multicolumn{2}{|l|}{ Charadriidae Leach, 1820} \\
\hline Vanellus cayanus (Latham, 1790) & batuíra-de-esporão \\
\hline Vanellus chilensis (Molina, 1782) & quero-quero \\
\hline Pluvialis dominica (Statius Muller, 1776) & batuiruçu \\
\hline Pluvialis squatarola (Linnaeus, 1758) & batuiruçu-de-axila-preta \\
\hline Charadrius semipalmatus Bonaparte, 1825 & batuíra-de-bando \\
\hline Charadrius wilsonia Ord, 1814 & batuíra-bicuda \\
\hline Charadrius collaris Vieillot, 1818 & batuíra-de-coleira \\
\hline Charadrius falklandicus Latham, 1790 & batuíra-de-coleira-dupla \\
\hline Charadrius modestus Lichtenstein, 1823 & batuíra-de-peito-tijolo \\
\hline \multicolumn{2}{|l|}{ Haematopodidae Bonaparte, 1838} \\
\hline Haematopus palliatus Temminck, 1820 & piru-piru \\
\hline \multicolumn{2}{|l|}{ Recurvirostridae Bonaparte, 1831} \\
\hline Himantopus melanurus Vieillot, 1817 & pernilongo-de-costas-brancas \\
\hline \multicolumn{2}{|l|}{ Scolopacidae Rafinesque, 1815} \\
\hline Gallinago paraguaiae (Vieillot, 1816) & narceja \\
\hline Gallinago undulata (Boddaert, 1783) & narcejão \\
\hline Limosa haemastica (Linnaeus, 1758) & maçarico-de-bico-virado \\
\hline Numenius borealis (Forster, 1772) & maçarico-esquimó \\
\hline Numenius phaeopus (Linnaeus, 1758) & maçarico-galego \\
\hline Bartramia longicauda (Bechstein, 1812) & maçarico-do-campo \\
\hline Actitis macularius (Linnaeus, 1766) & maçarico-pintado \\
\hline Tringa solitaria Wilson, 1813 & maçarico-solitário \\
\hline Tringa melanoleuca (Gmelin, 1789) & maçarico-grande-de-perna-amarela \\
\hline Tringa semipalmata (Gmelin, 1789) & maçarico-de-asa-branca \\
\hline Tringa flavipes (Gmelin, 1789) & maçarico-de-perna-amarela \\
\hline Arenaria interpres (Linnaeus, 1758) & vira-pedras \\
\hline Calidris canutus (Linnaeus, 1758) & maçarico-de-papo-vermelho \\
\hline Calidris alba (Pallas, 1764) & maçarico-branco \\
\hline Calidris pusilla (Linnaeus, 1766) & maçarico-rasteirinho \\
\hline Calidris minutilla (Vieillot, 1819) & maçariquinho \\
\hline Calidris fuscicollis (Vieillot, 1819) & maçarico-de-sobre-branco \\
\hline Calidris bairdii (Coues, 1861) & maçarico-de-bico-fino \\
\hline Calidris melanotos (Vieillot, 1819) & maçarico-de-colete \\
\hline Calidris himantopus (Bonaparte, 1826) & maçarico-pernilongo \\
\hline Tryngites subruficollis (Vieillot, 1819) & maçarico-acanelado \\
\hline Phalaropus tricolor (Vieillot, 1819) & pisa-n’água \\
\hline Jacanidae Chenu \& Des Murs, 1854 & \\
\hline
\end{tabular}


Tabela 1. Continuação...

\begin{tabular}{|c|c|}
\hline Nome do táxon & Nome em português \\
\hline Jacana jacana (Linnaeus, 1766) & jaçanã \\
\hline \multicolumn{2}{|l|}{ Rostratulidae Mathews, 1914} \\
\hline Nycticryphes semicollaris (Vieillot, 1816) & narceja-de-bico-torto \\
\hline \multicolumn{2}{|l|}{ Stercorariidae Gray, 1870} \\
\hline Stercorarius skua (Brünnich, 1764) & mandrião-grande \\
\hline Stercorarius maccormicki Saunders, 1893 & mandrião-do-sul \\
\hline Stercorarius pomarinus (Temminck, 1815) & mandrião-pomarino \\
\hline Stercorarius parasiticus (Linnaeus, 1758) & mandrião-parasítico \\
\hline \multicolumn{2}{|l|}{ Laridae Rafinesque, 1815} \\
\hline Chroicocephalus maculipennis (Lichtenstein, 1823) & gaivota-maria-velha \\
\hline Larus dominicanus Lichtenstein, 1823 & gaivotão \\
\hline \multicolumn{2}{|l|}{ Sternidae Vigors, 1825} \\
\hline Anous stolidus (Linnaeus, 1758) & trinta-réis-escuro \\
\hline Sternula superciliaris (Vieillot, 1819) & trinta-réis-anão \\
\hline Phaetusa simplex (Gmelin, 1789) & trinta-réis-grande \\
\hline Gelochelidon nilotica (Gmelin, 1789) & trinta-réis-de-bico-preto \\
\hline Sterna hirundo Linnaeus, 1758 & trinta-réis-boreal \\
\hline Sterna paradisaea Pontoppidan, 1763 & trinta-réis-ártico \\
\hline Sterna hirundinacea Lesson, 1831 & trinta-réis-de-bico-vermelho \\
\hline Sterna trudeaui Audubon, 1838 & trinta-réis-de-coroa-branca \\
\hline Thalasseus sandvicensis (Latham, 1787) & trinta-réis-de-bando \\
\hline Thalasseus maximus (Boddaert, 1783) & trinta-réis-real \\
\hline \multicolumn{2}{|l|}{ Rynchopidae Bonaparte, 1838} \\
\hline Rynchops niger Linnaeus, 1758 & talha-mar \\
\hline \multicolumn{2}{|l|}{ Columbiformes Latham, 1790} \\
\hline \multicolumn{2}{|l|}{ Columbidae Leach, 1820} \\
\hline Columbina minuta (Linnaeus, 1766) & rolinha-de-asa-canela \\
\hline Columbina talpacoti $($ Temminck, 1811) & rolinha-roxa \\
\hline Columbina squammata (Lesson, 1831) & fogo-apagou \\
\hline Columbina picui (Temminck, 1813) & rolinha-picui \\
\hline Columbina cyanopis (Pelzeln, 1870) & rolinha-do-planalto \\
\hline Claravis pretiosa (Ferrari-Perez, 1886) & pararu-azul \\
\hline Claravis godefrida (Temminck, 1811) & pararu-espelho \\
\hline Columba livia Gmelin, 1789 & pombo-doméstico \\
\hline Patagioenas speciosa (Gmelin, 1789) & pomba-trocal \\
\hline Patagioenas picazuro (Temminck, 1813) & pombão \\
\hline Patagioenas cayennensis (Bonnaterre, 1792) & pomba-galega \\
\hline Patagioenas plumbea (Vieillot, 1818) & pomba-amargosa \\
\hline Zenaida auriculata (Des Murs, 1847) & pomba-de-bando \\
\hline Leptotila verreauxi Bonaparte, 1855 & juriti-pupu \\
\hline Leptotila rufaxilla (Richard \& Bernard, 1792) & juriti-gemedeira \\
\hline Geotrygon violacea $($ Temminck, 1809$)$ & juriti-vermelha \\
\hline Geotrygon montana (Linnaeus, 1758) & pariri \\
\hline \multicolumn{2}{|l|}{ Psittaciformes Wagler, 1830} \\
\hline \multicolumn{2}{|l|}{ Psittacidae Rafinesque, 1815} \\
\hline Ara ararauna (Linnaeus, 1758) & arara-canindé \\
\hline Ara chloropterus Gray, 1859 & arara-vermelha-grande \\
\hline Orthopsittaca manilata (Boddaert, 1783) & maracanã-do-buriti \\
\hline Primolius maracana (Vieillot, 1816) & maracanã-verdadeira \\
\hline Diopsittaca nobilis (Linnaeus, 1758) & maracanã-pequena \\
\hline Aratinga leucophthalma (Statius Muller, 1776) & periquitão-maracanã \\
\hline
\end{tabular}


Tabela 1. Continuação...

\begin{tabular}{|c|c|}
\hline Nome do táxon & Nome em português \\
\hline Aratinga auricapillus (Kuhl, 1820) & jandaia-de-testa-vermelha \\
\hline Aratinga aurea (Gmelin, 1788) & periquito-rei \\
\hline Pyrrhura frontalis (Vieillot, 1817) & tiriba-de-testa-vermelha \\
\hline Myiopsitta monachus (Boddaert, 1783) & caturrita \\
\hline Forpus xanthopterygius (Spix, 1824) & tuim \\
\hline Brotogeris tirica (Gmelin, 1788) & periquito-rico \\
\hline Brotogeris chiriri (Vieillot, 1818) & periquito-de-encontro-amarelo \\
\hline Touit melanonotus (Wied, 1820) & apuim-de-costas-pretas \\
\hline Pionopsitta pileata (Scopoli, 1769) & cuiú-cuiú \\
\hline Alipiopsitta xanthops (Spix, 1824) & papagaio-galego \\
\hline Pionus maximiliani (Kuhl, 1820) & maitaca-verde \\
\hline Amazona vinacea (Kuhl, 1820) & papagaio-de-peito-roxo \\
\hline Amazona farinosa (Boddaert, 1783) & papagaio-moleiro \\
\hline Amazona brasiliensis (Linnaeus, 1758) & papagaio-de-cara-roxa \\
\hline Amazona amazonica (Linnaeus, 1766) & curica \\
\hline Amazona aestiva (Linnaeus, 1758) & papagaio-verdadeiro \\
\hline Triclaria malachitacea (Spix, 1824) & sabiá-cica \\
\hline \multicolumn{2}{|l|}{ Cuculiformes Wagler, 1830} \\
\hline \multicolumn{2}{|l|}{ Cuculidae Leach, 1820} \\
\hline Micrococcyx cinereus (Vieillot, 1817) & papa-lagarta-cinzento \\
\hline Piaya cayana (Linnaeus, 1766) & alma-de-gato \\
\hline Coccyzus melacoryphus Vieillot, 1817 & papa-lagarta-acanelado \\
\hline Coccyzus americanus (Linnaeus, 1758) & papa-lagarta-de-asa-vermelha \\
\hline Coccyzus euleri Cabanis, 1873 & papa-lagarta-de-euler \\
\hline Crotophaga major Gmelin, 1788 & anu-coroca \\
\hline Crotophaga ani Linnaeus, 1758 & anu-preto \\
\hline Guira guira (Gmelin, 1788) & anu-branco \\
\hline Tapera naevia (Linnaeus, 1766) & saci \\
\hline Dromococcyx phasianellus (Spix, 1824) & peixe-frito-verdadeiro \\
\hline Dromococcyx pavoninus Pelzeln, 1870 & peixe-frito-pavonino \\
\hline \multicolumn{2}{|l|}{ Strigiformes Wagler, 1830} \\
\hline \multicolumn{2}{|l|}{ Tytonidae Mathews, 1912} \\
\hline Tyto alba (Scopoli, 1769) & coruja-da-igreja \\
\hline \multicolumn{2}{|l|}{ Strigidae Leach, 1820} \\
\hline Megascops choliba (Vieillot, 1817) & corujinha-do-mato \\
\hline Megascops atricapilla (Temminck, 1822) & corujinha-sapo \\
\hline Pulsatrix perspicillata (Latham, 1790) & murucututu \\
\hline Pulsatrix koeniswaldiana (Bertoni \& Bertoni, 1901) & murucututu-de-barriga-amarela \\
\hline Bubo virginianus (Gmelin, 1788) & jacurutu \\
\hline Strix hylophila Temminck, 1825 & coruja-listrada \\
\hline Strix virgata (Cassin, 1849) & coruja-do-mato \\
\hline Strix huhula Daudin, 1800 & coruja-preta \\
\hline Glaucidium minutissimum (Wied, 1830) & caburé-miudinho \\
\hline Glaucidium brasilianum (Gmelin, 1788) & caburé \\
\hline Athene cunicularia (Molina, 1782) & coruja-buraqueira \\
\hline Aegolius harrisii (Cassin, 1849) & caburé-acanelado \\
\hline Asio clamator (Vieillot, 1808) & coruja-orelhuda \\
\hline Asio stygius (Wagler, 1832) & mocho-diabo \\
\hline Asio flammeus (Pontoppidan, 1763) & mocho-dos-banhados \\
\hline \multicolumn{2}{|l|}{ Caprimulgiformes Ridgway, 1881} \\
\hline Nyctibiidae Chenu \& Des Murs, 1851 & \\
\hline
\end{tabular}


Tabela 1. Continuação...

\begin{tabular}{|c|c|}
\hline Nome do táxon & Nome em português \\
\hline Nyctibius grandis (Gmelin, 1789) & mãe-da-lua-gigante \\
\hline Nyctibius aethereus (Wied, 1820) & mãe-da-lua-parda \\
\hline Nyctibius griseus (Gmelin, 1789) & mãe-da-lua \\
\hline \multicolumn{2}{|l|}{ Caprimulgidae Vigors, 1825} \\
\hline Lurocalis semitorquatus (Gmelin, 1789) & tuju \\
\hline Chordeiles acutipennis (Hermann, 1783) & bacurau-de-asa-fina \\
\hline Chordeiles minor (Forster, 1771) & bacurau-norte-americano \\
\hline Podager nacunda (Vieillot, 1817) & corucão \\
\hline Nyctidromus albicollis (Gmelin, 1789) & bacurau \\
\hline Nyctiphrynus ocellatus (Tschudi, 1844) & bacurau-ocelado \\
\hline Caprimulgus rufus Boddaert, 1783 & joão-corta-pau \\
\hline Caprimulgus sericocaudatus (Cassin, 1849) & bacurau-rabo-de-seda \\
\hline Caprimulgus longirostris Bonaparte, 1825 & bacurau-da-telha \\
\hline Caprimulgus maculicaudus (Lawrence, 1862) & bacurau-de-rabo-maculado \\
\hline Caprimulgus parvulus Gould, 1837 & bacurau-chintã \\
\hline Hydropsalis torquata (Gmelin, 1789) & bacurau-tesoura \\
\hline Macropsalis forcipata (Nitzsch, 1840) & bacurau-tesoura-gigante \\
\hline Eleothreptus anomalus (Gould, 1838) & curiango-do-banhado \\
\hline Eleothreptus candicans (Pelzeln, 1867) & bacurau-de-rabo-branco \\
\hline \multicolumn{2}{|l|}{ Apodiformes Peters, 1940} \\
\hline \multicolumn{2}{|l|}{ Apodidae Olphe-Galliard, 1887} \\
\hline Cypseloides fumigatus (Streubel, 1848) & taperuçu-preto \\
\hline Cypseloides senex (Temminck, 1826) & taperuçu-velho \\
\hline Streptoprocne zonaris (Shaw, 1796) & taperuçu-de-coleira-branca \\
\hline Streptoprocne biscutata (Sclater, 1866) & taperuçu-de-coleira-falha \\
\hline Chaetura cinereiventris Sclater, 1862 & andorinhão-de-sobre-cinzento \\
\hline Chaetura meridionalis Hellmayr, 1907 & andorinhão-do-temporal \\
\hline Tachornis squamata (Cassin, 1853) & tesourinha \\
\hline Panyptila cayennensis (Gmelin, 1789) & andorinhão-estofador \\
\hline \multicolumn{2}{|l|}{ Trochilidae Vigors, 1825} \\
\hline Ramphodon naevius (Dumont, 1818) & beija-flor-rajado \\
\hline Glaucis hirsutus (Gmelin, 1788) & balança-rabo-de-bico-torto \\
\hline Phaethornis squalidus (Temminck, 1822) & rabo-branco-pequeno \\
\hline Phaethornis ruber (Linnaeus, 1758) & rabo-branco-rubro \\
\hline Phaethornis pretrei (Lesson \& Delattre, 1839) & rabo-branco-acanelado \\
\hline Phaethornis eurynome (Lesson, 1832) & rabo-branco-de-garganta-rajada \\
\hline Eupetomena macroura (Gmelin, 1788) & beija-flor-tesoura \\
\hline Aphantochroa cirrochloris (Vieillot, 1818) & beija-flor-cinza \\
\hline Florisuga fusca (Vieillot, 1817) & beija-flor-preto \\
\hline Colibri serrirostris (Vieillot, 1816) & beija-flor-de-orelha-violeta \\
\hline Anthracothorax nigricollis (Vieillot, 1817) & beija-flor-de-veste-preta \\
\hline Chrysolampis mosquitus (Linnaeus, 1758) & beija-flor-vermelho \\
\hline Stephanoxis lalandi (Vieillot, 1818) & beija-flor-de-topete \\
\hline Lophornis magnificus (Vieillot, 1817) & topetinho-vermelho \\
\hline Lophornis chalybeus (Vieillot, 1822) & topetinho-verde \\
\hline Chlorostilbon lucidus (Shaw, 1812) & besourinho-de-bico-vermelho \\
\hline Thalurania furcata (Gmelin, 1788) & beija-flor-tesoura-verde \\
\hline Thalurania glaucopis (Gmelin, 1788) & beija-flor-de-fronte-violeta \\
\hline Hylocharis sapphirina (Gmelin, 1788) & beija-flor-safira \\
\hline Hylocharis cyanus (Vieillot, 1818) & beija-flor-roxo \\
\hline Hylocharis chrysura (Shaw, 1812) & beija-flor-dourado \\
\hline
\end{tabular}


Tabela 1. Continuação...

\begin{tabular}{|c|c|}
\hline Nome do táxon & Nome em português \\
\hline Leucochloris albicollis (Vieillot, 1818) & beija-flor-de-papo-branco \\
\hline Polytmus guainumbi (Pallas, 1764) & beija-flor-de-bico-curvo \\
\hline Amazilia versicolor (Vieillot, 1818) & beija-flor-de-banda-branca \\
\hline Amazilia brevirostris (Lesson, 1829) & beija-flor-de-bico-preto \\
\hline Amazilia fimbriata (Gmelin, 1788) & beija-flor-de-garganta-verde \\
\hline Amazilia lactea (Lesson, 1832) & beija-flor-de-peito-azul \\
\hline Clytolaema rubricauda (Boddaert, 1783) & beija-flor-rubi \\
\hline Heliothryx auritus (Gmelin, 1788) & beija-flor-de-bochecha-azul \\
\hline Heliactin bilophus (Temminck, 1820) & chifre-de-ouro \\
\hline Heliomaster longirostris (Audebert \& Vieillot, 1801) & bico-reto-cinzento \\
\hline Heliomaster squamosus (Temminck, 1823) & bico-reto-de-banda-branca \\
\hline Calliphlox amethystina (Boddaert, 1783) & estrelinha-ametista \\
\hline \multicolumn{2}{|l|}{ Trogoniformes A. O. U., 1886} \\
\hline \multicolumn{2}{|l|}{ Trogonidae Lesson, 1828} \\
\hline Trogon viridis Linnaeus, 1766 & surucuá-grande-de-barriga-amarela \\
\hline Trogon surrucura Vieillot, 1817 & surucuá-variado \\
\hline Trogon rufus Gmelin, 1788 & surucuá-de-barriga-amarela \\
\hline \multicolumn{2}{|l|}{ Coraciiformes Forbes, 1844} \\
\hline \multicolumn{2}{|l|}{ Alcedinidae Rafinesque, 1815} \\
\hline Megaceryle torquata (Linnaeus, 1766) & martim-pescador-grande \\
\hline Chloroceryle amazona (Latham, 1790) & martim-pescador-verde \\
\hline Chloroceryle aenea (Pallas, 1764) & martinho \\
\hline Chloroceryle americana (Gmelin, 1788) & martim-pescador-pequeno \\
\hline Chloroceryle inda (Linnaeus, 1766) & martim-pescador-da-mata \\
\hline \multicolumn{2}{|l|}{ Momotidae Gray, 1840} \\
\hline Baryphthengus ruficapillus (Vieillot, 1818) & juruva-verde \\
\hline Momotus momota (Linnaeus, 1766) & udu-de-coroa-azul \\
\hline \multicolumn{2}{|l|}{ Galbuliformes Fürbringer, 1888} \\
\hline \multicolumn{2}{|l|}{ Galbulidae Vigors, 1825} \\
\hline Brachygalba lugubris (Swainson, 1838) & ariramba-preta \\
\hline Jacamaralcyon tridactyla (Vieillot, 1817) & cuitelão \\
\hline Galbula ruficauda Cuvier, 1816 & ariramba-de-cauda-ruiva \\
\hline \multicolumn{2}{|l|}{ Bucconidae Horsfield, 1821} \\
\hline Notharchus swainsoni (Gray, 1846) & macuru-de-barriga-castanha \\
\hline Nystalus chacuru (Vieillot, 1816) & joão-bobo \\
\hline Nystalus maculatus (Gmelin, 1788) & rapazinho-dos-velhos \\
\hline Malacoptila striata (Spix, 1824) & barbudo-rajado \\
\hline Nonnula rubecula (Spix, 1824) & macuru \\
\hline Monasa nigrifrons (Spix, 1824) & chora-chuva-preto \\
\hline Chelidoptera tenebrosa (Pallas, 1782) & urubuzinho \\
\hline \multicolumn{2}{|l|}{ Piciformes Meyer \& Wolf, 1810} \\
\hline \multicolumn{2}{|l|}{ Ramphastidae Vigors, 1825} \\
\hline Ramphastos toco Statius Muller, 1776 & tucanuçu \\
\hline Ramphastos vitellinus Lichtenstein, 1823 & tucano-de-bico-preto \\
\hline Ramphastos dicolorus Linnaeus, 1766 & tucano-de-bico-verde \\
\hline Selenidera maculirostris (Lichtenstein, 1823) & araçari-poca \\
\hline Pteroglossus bailloni (Vieillot, 1819) & araçari-banana \\
\hline Pteroglossus aracari (Linnaeus, 1758) & araçari-de-bico-branco \\
\hline Pteroglossus castanotis Gould, 1834 & araçari-castanho \\
\hline \multicolumn{2}{|l|}{ Picidae Leach, 1820} \\
\hline Picumnus cirratus Temminck, 1825 & pica-pau-anão-barrado \\
\hline
\end{tabular}


Tabela 1. Continuação...

\begin{tabular}{|c|c|}
\hline Nome do táxon & Nome em português \\
\hline Picumnus temminckii Lafresnaye, 1845 & pica-pau-anão-de-coleira \\
\hline Picumnus albosquamatus d'Orbigny, 1840 & pica-pau-anão-escamado \\
\hline Melanerpes candidus (Otto, 1796) & birro, pica-pau-branco \\
\hline Melanerpes flavifrons (Vieillot, 1818) & benedito-de-testa-amarela \\
\hline Veniliornis maculifrons (Spix, 1824) & picapauzinho-de-testa-pintada \\
\hline Veniliornis passerinus (Linnaeus, 1766) & picapauzinho-anão \\
\hline Veniliornis spilogaster (Wagler, 1827) & picapauzinho-verde-carijó \\
\hline Veniliornis mixtus (Boddaert, 1783) & pica-pau-chorão \\
\hline Piculus flavigula (Boddaert, 1783) & pica-pau-bufador \\
\hline Piculus aurulentus (Temminck, 1821) & pica-pau-dourado \\
\hline Colaptes melanochloros (Gmelin, 1788) & pica-pau-verde-barrado \\
\hline Colaptes campestris (Vieillot, 1818) & pica-pau-do-campo \\
\hline Celeus flavescens (Gmelin, 1788) & pica-pau-de-cabeça-amarela \\
\hline Dryocopus galeatus (Temminck, 1822) & pica-pau-de-cara-canela \\
\hline Dryocopus lineatus (Linnaeus, 1766) & pica-pau-de-banda-branca \\
\hline Campephilus robustus (Lichtenstein, 1818) & pica-pau-rei \\
\hline Campephilus melanoleucos (Gmelin, 1788) & pica-pau-de-topete-vermelho \\
\hline \multicolumn{2}{|l|}{ Passeriformes Linnaeus, 1758} \\
\hline \multicolumn{2}{|c|}{ Melanopareiidae Irestedt, Fjeldså, Johansson \& Ericson, 2002} \\
\hline Melanopareia torquata (Wied, 1831) & tapaculo-de-colarinho \\
\hline \multicolumn{2}{|l|}{ Thamnophilidae Swainson, 1824} \\
\hline Hypoedaleus guttatus (Vieillot, 1816) & chocão-carijó \\
\hline Batara cinerea (Vieillot, 1819) & matracão \\
\hline Mackenziaena leachii (Such, 1825) & borralhara-assobiadora \\
\hline Mackenziaena severa (Lichtenstein, 1823) & borralhara \\
\hline Taraba major (Vieillot, 1816) & choró-boi \\
\hline Biatas nigropectus (Lafresnaye, 1850) & papo-branco \\
\hline Thamnophilus doliatus (Linnaeus, 1764) & choca-barrada \\
\hline Thamnophilus ruficapillus Vieillot, 1816 & choca-de-chapéu-vermelho \\
\hline Thamnophilus torquatus Swainson, 1825 & choca-de-asa-vermelha \\
\hline Thamnophilus palliatus (Lichtenstein, 1823) & choca-listrada \\
\hline Thamnophilus pelzelni Hellmayr, 1924 & choca-do-planalto \\
\hline Thamnophilus caerulescens Vieillot, 1816 & choca-da-mata \\
\hline Dysithamnus stictothorax (Temminck, 1823) & choquinha-de-peito-pintado \\
\hline Dysithamnus mentalis (Temminck, 1823) & choquinha-lisa \\
\hline Dysithamnus xanthopterus Burmeister, 1856 & choquinha-de-asa-ferrugem \\
\hline Myrmotherula gularis (Spix, 1825) & choquinha-de-garganta-pintada \\
\hline Myrmotherula minor Salvadori, 1864 & choquinha-pequena \\
\hline Myrmotherula unicolor (Ménétriès, 1835) & choquinha-cinzenta \\
\hline Herpsilochmus atricapillus Pelzeln, 1868 & chorozinho-de-chapéu-preto \\
\hline Herpsilochmus longirostris Pelzeln, 1868 & chorozinho-de-bico-comprido \\
\hline Herpsilochmus rufimarginatus (Temminck, 1822) & chorozinho-de-asa-vermelha \\
\hline Formicivora melanogaster Pelzeln, 1868 & formigueiro-de-barriga-preta \\
\hline Formicivora rufa (Wied, 1831) & papa-formiga-vermelho \\
\hline Formicivora aff. acutirostris & bicudinho-do-brejo-paulista \\
\hline Drymophila ferruginea (Temminck, 1822) & trovoada \\
\hline Drymophila rubricollis (Bertoni, 1901) & trovoada-de-bertoni \\
\hline Drymophila genei (Filippi, 1847) & choquinha-da-serra \\
\hline Drymophila ochropyga (Hellmayr, 1906) & choquinha-de-dorso-vermelho \\
\hline Drymophila malura (Temminck, 1825) & choquinha-carijó \\
\hline Drymophila squamata (Lichtenstein, 1823) & pintadinho \\
\hline
\end{tabular}


Tabela 1. Continuação...

\begin{tabular}{|c|c|}
\hline Nome do táxon & Nome em português \\
\hline Terenura maculata (Wied, 1831) & zidedê \\
\hline Pyriglena leucoptera (Vieillot, 1818) & papa-taoca-do-sul \\
\hline Myrmeciza loricata (Lichtenstein, 1823) & formigueiro-assobiador \\
\hline Myrmeciza squamosa Pelzeln, 1868 & papa-formiga-de-grota \\
\hline \multicolumn{2}{|l|}{ Conopophagidae Sclater \& Salvin, 1873} \\
\hline Conopophaga lineata (Wied, 1831) & chupa-dente \\
\hline Conopophaga melanops (Vieillot, 1818) & cuspidor-de-máscara-preta \\
\hline \multicolumn{2}{|l|}{ Grallariidae Sclater \& Salvin, 1873} \\
\hline Grallaria varia (Boddaert, 1783) & tovacuçu \\
\hline Hylopezus nattereri (Pinto, 1937) & pinto-do-mato \\
\hline \multicolumn{2}{|l|}{ Rhinocryptidae Wetmore, 1930} \\
\hline Psilorhamphus guttatus (Ménétriès, 1835) & tapaculo-pintado \\
\hline Merulaxis ater Lesson, 1830 & entufado \\
\hline Eleoscytalopus indigoticus (Wied, 1831) & macuquinho \\
\hline Scytalopus speluncae (Ménétriès, 1835). & tapaculo-preto \\
\hline \multicolumn{2}{|l|}{ Formicariidae Gray, 1840} \\
\hline Formicarius colma Boddaert, 1783 & galinha-do-mato \\
\hline Chamaeza campanisona (Lichtenstein, 1823) & tovaca-campainha \\
\hline Chamaeza meruloides Vigors, 1825 & tovaca-cantadora \\
\hline Chamaeza ruficauda (Cabanis \& Heine, 1859) & tovaca-de-rabo-vermelho \\
\hline \multicolumn{2}{|l|}{ Scleruridae Swainson, 1827} \\
\hline Sclerurus mexicanus Sclater, 1857 & vira-folha-de-peito-vermelho \\
\hline Sclerurus scansor (Ménétriès, 1835) & vira-folha \\
\hline Geositta poeciloptera (Wied, 1830) & andarilho \\
\hline \multicolumn{2}{|l|}{ Dendrocolaptidae Gray, 1840} \\
\hline Dendrocincla turdina (Lichtenstein, 1820) & arapaçu-liso \\
\hline Sittasomus griseicapillus (Vieillot, 1818) & arapaçu-verde \\
\hline Xiphocolaptes albicollis (Vieillot, 1818) & arapaçu-de-garganta-branca \\
\hline Dendrocolaptes platyrostris Spix, 1825 & arapaçu-grande \\
\hline Xiphorhynchus fuscus (Vieillot, 1818) & arapaçu-rajado \\
\hline Lepidocolaptes angustirostris (Vieillot, 1818) & arapaçu-de-cerrado \\
\hline Lepidocolaptes squamatus (Lichtenstein, 1822) & arapaçu-escamado \\
\hline Lepidocolaptes falcinellus (Cabanis \& Heine, 1859) & arapaçu-escamado-do-sul \\
\hline Campylorhamphus trochilirostris (Lichtenstein, 1820) & arapaçu-beija-flor \\
\hline Campylorhamphus falcularius (Vieillot, 1822) & arapaçu-de-bico-torto \\
\hline \multicolumn{2}{|l|}{ Furnariidae Gray, 1840} \\
\hline Furnarius figulus (Lichtenstein, 1823) & casaca-de-couro-da-lama \\
\hline Furnarius rufus (Gmelin, 1788) & joão-de-barro \\
\hline Phleocryptes melanops (Vieillot, 1817) & bate-bico \\
\hline Leptasthenura setaria (Temminck, 1824) & grimpeiro \\
\hline Oreophylax moreirae (Miranda-Ribeiro, 1906) & garrincha-chorona \\
\hline Schoeniophylax phryganophilus (Vieillot, 1817) & bichoita \\
\hline Synallaxis ruficapilla Vieillot, 1819 & pichororé \\
\hline Synallaxis cinerascens Temminck, 1823 & pi-puí \\
\hline Synallaxis frontalis Pelzeln, 1859 & petrim \\
\hline Synallaxis albescens Temminck, 1823 & uí-pi \\
\hline Synallaxis spixi Sclater, 1856 & joão-teneném \\
\hline Synallaxis hypospodia Sclater, 1874 & joão-grilo \\
\hline Synallaxis scutata Sclater, 1859 & estrelinha-preta \\
\hline Cranioleuca vulpina (Pelzeln, 1856) & arredio-do-rio \\
\hline Cranioleuca obsoleta (Reichenbach, 1853) & arredio-oliváceo \\
\hline
\end{tabular}


Tabela 1. Continuação...

\begin{tabular}{|c|c|}
\hline Nome do táxon & Nome em português \\
\hline Cranioleuca pallida (Wied, 1831) & arredio-pálido \\
\hline Certhiaxis cinnamomeus (Gmelin, 1788) & curutié \\
\hline Phacellodomus rufifrons (Wied, 1821) & joão-de-pau \\
\hline Phacellodomus ruber (Vieillot, 1817) & graveteiro \\
\hline Phacellodomus erythrophthalmus (Wied, 1821) & joão-botina-da-mata \\
\hline Phacellodomus ferrugineigula (Pelzeln, 1858) & joão-botina-do-brejo \\
\hline Clibanornis dendrocolaptoides (Pelzeln, 1859) & cisqueiro \\
\hline Anumbius annumbi (Vieillot, 1817) & cochicho \\
\hline Anabacerthia amaurotis (Temminck, 1823) & limpa-folha-miúdo \\
\hline Syndactyla rufosuperciliata (Lafresnaye, 1832) & trepador-quiete \\
\hline Philydor lichtensteini Cabanis \& Heine, 1859 & limpa-folha-ocráceo \\
\hline Philydor atricapillus (Wied, 1821) & limpa-folha-coroado \\
\hline Philydor rufum (Vieillot, 1818) & limpa-folha-de-testa-baia \\
\hline Anabazenops fuscus (Vieillot, 1816) & trepador-coleira \\
\hline Cichlocolaptes leucophrus (Jardine \& Selby, 1830) & trepador-sobrancelha \\
\hline Automolus leucophthalmus (Wied, 1821) & barranqueiro-de-olho-branco \\
\hline Hylocryptus rectirostris (Wied, 1831) & fura-barreira \\
\hline Lochmias nematura (Lichtenstein, 1823) & joão-porca \\
\hline Heliobletus contaminatus Berlepsch, 1885 & trepadorzinho \\
\hline Xenops minutus (Sparrman, 1788) & bico-virado-miúdo \\
\hline Xenops rutilans Temminck, 1821 & bico-virado-carijó \\
\hline \multicolumn{2}{|l|}{ Tyrannidae Vigors, 1825} \\
\hline Mionectes rufiventris Cabanis, 1846 & abre-asa-de-cabeça-cinza \\
\hline Leptopogon amaurocephalus Tschudi, 1846 & cabeçudo \\
\hline Corythopis delalandi (Lesson, 1830) & estalador \\
\hline Hemitriccus diops (Temminck, 1822) & olho-falso \\
\hline Hemitriccus obsoletus (Miranda-Ribeiro, 1906) & catraca \\
\hline Hemitriccus orbitatus (Wied, 1831) & tiririzinho-do-mato \\
\hline Hemitriccus nidipendulus (Wied, 1831) & tachuri-campainha \\
\hline Hemitriccus margaritaceiventer (d'Orbigny \& Lafresnaye, 1837) & sebinho-de-olho-de-ouro \\
\hline Hemitriccus furcatus (Lafresnaye, 1846) & papa-moscas-estrela \\
\hline Myiornis auricularis (Vieillot, 1818) & miudinho \\
\hline Poecilotriccus plumbeiceps (Lafresnaye, 1846) & tororó \\
\hline Poecilotriccus latirostris (Pelzeln, 1868) & ferreirinho-de-cara-parda \\
\hline Todirostrum poliocephalum (Wied, 1831) & teque-teque \\
\hline Todirostrum cinereum (Linnaeus, 1766) & ferreirinho-relógio \\
\hline Phyllomyias burmeisteri Cabanis \& Heine, 1859 & piolhinho-chiador \\
\hline Phyllomyias virescens (Temminck, 1824) & piolhinho-verdoso \\
\hline Phyllomyias fasciatus (Thunberg, 1822) & piolhinho \\
\hline Phyllomyias griseocapilla Sclater, 1862 & piolhinho-serrano \\
\hline Myiopagis gaimardii (d'Orbigny, 1839) & maria-pechim \\
\hline Myiopagis caniceps (Swainson, 1835) & guaracava-cinzenta \\
\hline Myiopagis viridicata (Vieillot, 1817) & guaracava-de-crista-alaranjada \\
\hline Elaenia flavogaster (Thunberg, 1822) & guaracava-de-barriga-amarela \\
\hline Elaenia spectabilis Pelzeln, 1868 & guaracava-grande \\
\hline Elaenia chilensis Hellmayr, 1927 & guaracava-de-crista-branca \\
\hline Elaenia parvirostris Pelzeln, 1868 & guaracava-de-bico-curto \\
\hline Elaenia mesoleuca (Deppe, 1830) & tuque \\
\hline Elaenia cristata Pelzeln, 1868 & guaracava-de-topete-uniforme \\
\hline Elaenia chiriquensis Lawrence, 1865 & chibum \\
\hline Elaenia obscura (d'Orbigny \& Lafresnaye, 1837) & tucão \\
\hline
\end{tabular}


Tabela 1. Continuação...

\begin{tabular}{|c|c|}
\hline Nome do táxon & Nome em português \\
\hline Camptostoma obsoletum (Temminck, 1824) & risadinha \\
\hline Suiriri suiriri (Vieillot, 1818) & suiriri-cinzento \\
\hline Serpophaga nigricans (Vieillot, 1817) & joão-pobre \\
\hline Serpophaga subcristata (Vieillot, 1817) & alegrinho \\
\hline Phaeomyias murina (Spix, 1825) & bagageiro \\
\hline Capsiempis flaveola (Lichtenstein, 1823) & marianinha-amarela \\
\hline Polystictus pectoralis (Vieillot, 1817) & papa-moscas-canela \\
\hline Polystictus superciliaris (Wied, 1831) & papa-moscas-de-costas-cinzentas \\
\hline Pseudocolopteryx sclateri (Oustalet, 1892) & tricolino \\
\hline Pseudocolopteryx flaviventris (d'Orbigny \& Lafresnaye, 1837) & amarelinho-do-junco \\
\hline Euscarthmus meloryphus Wied, 1831 & barulhento \\
\hline Euscarthmus rufomarginatus (Pelzeln, 1868) & maria-corruíra \\
\hline Phylloscartes eximius (Temminck, 1822) & barbudinho \\
\hline Phylloscartes ventralis (Temminck, 1824) & borboletinha-do-mato \\
\hline Phylloscartes kronei Willis \& Oniki, 1992 & maria-da-restinga \\
\hline Phylloscartes paulista Ihering \& Ihering, 1907 & não-pode-parar \\
\hline Phylloscartes oustaleti (Sclater, 1887) & papa-moscas-de-olheiras \\
\hline Phylloscartes difficilis (Ihering \& Ihering, 1907) & estalinho \\
\hline Phylloscartes sylviolus (Cabanis \& Heine, 1859) & maria-pequena \\
\hline Sublegatus modestus (Wied, 1831) & guaracava-modesta \\
\hline Tachuris rubrigastra (Vieillot, 1817) & papa-piri \\
\hline Culicivora caudacuta (Vieillot, 1818) & papa-moscas-do-campo \\
\hline Tolmomyias sulphurescens (Spix, 1825) & bico-chato-de-orelha-preta \\
\hline Platyrinchus mystaceus Vieillot, 1818 & patinho \\
\hline Platyrinchus leucoryphus Wied, 1831 & patinho-gigante \\
\hline Onychorhynchus swainsoni (Pelzeln, 1858) & maria-leque-do-sudeste \\
\hline Myiophobus fasciatus (Statius Muller, 1776) & filipe \\
\hline Myiobius barbatus (Gmelin, 1789) & assanhadinho \\
\hline Myiobius atricaudus Lawrence, 1863 & assanhadinho-de-cauda-preta \\
\hline Hirundinea ferruginea (Gmelin, 1788) & gibão-de-couro \\
\hline Lathrotriccus euleri (Cabanis, 1868) & enferrujado \\
\hline Cnemotriccus fuscatus (Wied, 1831) & guaracavuçu \\
\hline Contopus cooperi (Nuttall, 1831) & piui-boreal \\
\hline Contopus virens (Linnaeus, 1766) & piui-verdadeiro \\
\hline Contopus cinereus (Spix, 1825) & papa-moscas-cinzento \\
\hline Pyrocephalus rubinus (Boddaert, 1783) & príncipe \\
\hline Lessonia rufa (Gmelin, 1789) & colegial \\
\hline Knipolegus cyanirostris (Vieillot, 1818) & maria-preta-de-bico-azulado \\
\hline Knipolegus lophotes Boie, 1828 & maria-preta-de-penacho \\
\hline Knipolegus nigerrimus (Vieillot, 1818) & maria-preta-de-garganta-vermelha \\
\hline Hymenops perspicillatus (Gmelin, 1789) & viuvinha-de-óculos \\
\hline Satrapa icterophrys (Vieillot, 1818) & suiriri-pequeno \\
\hline Xolmis cinereus (Vieillot, 1816) & primavera \\
\hline Xolmis velatus (Lichtenstein, 1823) & noivinha-branca \\
\hline Gubernetes yetapa (Vieillot, 1818) & tesoura-do-brejo \\
\hline Muscipipra vetula (Lichtenstein, 1823) & tesoura-cinzenta \\
\hline Fluvicola albiventer (Spix, 1825) & lavadeira-de-cara-branca \\
\hline Fluvicola nengeta (Linnaeus, 1766) & lavadeira-mascarada \\
\hline Arundinicola leucocephala (Linnaeus, 1764) & freirinha \\
\hline Alectrurus tricolor (Vieillot, 1816) & galito \\
\hline Colonia colonus (Vieillot, 1818) & viuvinha \\
\hline
\end{tabular}


Tabela 1. Continuação...

\begin{tabular}{|c|c|}
\hline Nome do táxon & Nome em português \\
\hline Machetornis rixosa (Vieillot, 1819) & suiriri-cavaleiro \\
\hline Legatus leucophaius (Vieillot, 1818) & bem-te-vi-pirata \\
\hline Myiozetetes cayanensis (Linnaeus, 1766) & bentevizinho-de-asa-ferrugínea \\
\hline Myiozetetes similis (Spix, 1825) & bentevizinho-de-penacho-vermelho \\
\hline Pitangus sulphuratus (Linnaeus, 1766) & bem-te-vi \\
\hline Philohydor lictor (Lichtenstein, 1823) & bentevizinho-do-brejo \\
\hline Conopias trivirgatus (Wied, 1831) & bem-te-vi-pequeno \\
\hline Myiodynastes maculatus (Statius Muller, 1776) & bem-te-vi-rajado \\
\hline Megarynchus pitangua (Linnaeus, 1766) & neinei \\
\hline Empidonomus varius (Vieillot, 1818) & peitica \\
\hline Griseotyrannus aurantioatrocristatus (d'Orbigny \& Lafresnaye, 1837) & peitica-de-chapéu-preto \\
\hline Tyrannus albogularis Burmeister, 1856 & suiriri-de-garganta-branca \\
\hline Tyrannus melancholicus Vieillot, 1819 & suiriri \\
\hline Tyrannus savana Vieillot, 1808 & tesourinha \\
\hline Tyrannus tyrannus (Linnaeus, 1766) & suiriri-valente \\
\hline Rhytipterna simplex (Lichtenstein, 1823) & vissiá \\
\hline Sirystes sibilator (Vieillot, 1818) & gritador \\
\hline Casiornis rufus (Vieillot, 1816) & caneleiro \\
\hline Myiarchus swainsoni Cabanis \& Heine, 1859 & irré \\
\hline Myiarchus ferox (Gmelin, 1789) & maria-cavaleira \\
\hline Myiarchus tyrannulus (Statius Muller, 1776) & maria-cavaleira-de-rabo-enferrujado \\
\hline Ramphotrigon megacephalum (Swainson, 1835) & maria-cabeçuda \\
\hline Attila phoenicurus Pelzeln, 1868 & capitão-castanho \\
\hline Attila rufus (Vieillot, 1819) & capitão-de-saíra \\
\hline \multicolumn{2}{|l|}{ Cotingidae Bonaparte, 1849} \\
\hline Phibalura flavirostris Vieillot, 1816 & tesourinha-da-mata \\
\hline Carpornis cucullata (Swainson, 1821) & corocochó \\
\hline Carpornis melanocephala (Wied, 1820) & sabiá-pimenta \\
\hline Procnias nudicollis (Vieillot, 1817) & araponga \\
\hline Tijuca atra Ferrusac, 1829 & saudade \\
\hline Lipaugus lanioides (Lesson, 1844) & tropeiro-da-serra \\
\hline Pyroderus scutatus (Shaw, 1792) & pavó \\
\hline \multicolumn{2}{|l|}{ Pipridae Rafinesque, 1815} \\
\hline Neopelma pallescens (Lafresnaye, 1853) & fruxu-do-cerradão \\
\hline Neopelma chrysolophum Pinto, 1944 & fruxu \\
\hline Piprites chloris (Temminck, 1822) & papinho-amarelo \\
\hline Piprites pileata $($ Temminck, 1822) & caneleirinho-de-chapéu-preto \\
\hline Ilicura militaris (Shaw \& Nodder, 1809) & tangarazinho \\
\hline Manacus manacus (Linnaeus, 1766) & rendeira \\
\hline Antilophia galeata (Lichtenstein, 1823) & soldadinho \\
\hline Chiroxiphia caudata (Shaw \& Nodder, 1793) & tangará \\
\hline Pipra fasciicauda Hellmayr, 1906 & uirapuru-laranja \\
\hline \multicolumn{2}{|l|}{ Tityridae Gray, 1840} \\
\hline Oxyruncus cristatus Swainson, 1821 & araponga-do-horto \\
\hline Schiffornis virescens (Lafresnaye, 1838) & flautim \\
\hline Laniisoma elegans (Thunberg, 1823) & chibante \\
\hline Iodopleura pipra (Lesson, 1831) & anambezinho \\
\hline Tityra inquisitor (Lichtenstein, 1823) & anambé-branco-de-bochecha-parda \\
\hline Tityra cayana (Linnaeus, 1766) & anambé-branco-de-rabo-preto \\
\hline Pachyramphus viridis (Vieillot, 1816) & caneleiro-verde \\
\hline
\end{tabular}


Tabela 1. Continuação...

\begin{tabular}{|c|c|}
\hline Nome do táxon & Nome em português \\
\hline Pachyramphus castaneus (Jardine \& Selby, 1827) & caneleiro \\
\hline Pachyramphus polychopterus (Vieillot, 1818) & caneleiro-preto \\
\hline Pachyramphus marginatus (Lichtenstein, 1823) & caneleiro-bordado \\
\hline Pachyramphus validus (Lichtenstein, 1823) & caneleiro-de-chapéu-preto \\
\hline \multicolumn{2}{|l|}{ Vireonidae Swainson, 1837} \\
\hline Cyclarhis gujanensis (Gmelin, 1789) & pitiguari \\
\hline Vireo olivaceus (Linnaeus, 1766) & juruviara \\
\hline Hylophilus poicilotis Temminck, 1822 & verdinho-coroado \\
\hline Hylophilus amaurocephalus (Nordmann, 1835) & vite-vite-de-olho-cinza \\
\hline Hylophilus thoracicus Temminck, 1822 & vite-vite \\
\hline \multicolumn{2}{|l|}{ Corvidae Leach, 1820} \\
\hline Cyanocorax cyanomelas (Vieillot, 1818) & gralha-do-pantanal \\
\hline Cyanocorax caeruleus (Vieillot, 1818) & gralha-azul \\
\hline Cyanocorax cristatellus (Temminck, 1823) & gralha-do-campo \\
\hline Cyanocorax chrysops (Vieillot, 1818) & gralha-picaça \\
\hline \multicolumn{2}{|l|}{ Hirundinidae Rafinesque, 1815} \\
\hline Pygochelidon cyanoleuca (Vieillot, 1817) & andorinha-pequena-de-casa \\
\hline Alopochelidon fucata (Temminck, 1822) & andorinha-morena \\
\hline Atticora tibialis (Cassin, 1853) & calcinha-branca \\
\hline Stelgidopteryx ruficollis (Vieillot, 1817) & andorinha-serradora \\
\hline Progne tapera (Vieillot, 1817) & andorinha-do-campo \\
\hline Progne subis (Linnaeus, 1758) & andorinha-azul \\
\hline Progne chalybea (Gmelin, 1789) & andorinha-doméstica-grande \\
\hline Tachycineta albiventer (Boddaert, 1783) & andorinha-do-rio \\
\hline Tachycineta leucorrhoa (Vieillot, 1817) & andorinha-de-sobre-branco \\
\hline Riparia riparia (Linnaeus, 1758) & andorinha-do-barranco \\
\hline Hirundo rustica Linnaeus, 1758 & andorinha-de-bando \\
\hline Petrochelidon pyrrhonota (Vieillot, 1817) & andorinha-de-dorso-acanelado \\
\hline \multicolumn{2}{|l|}{ Troglodytidae Swainson, 1831} \\
\hline Troglodytes musculus Naumann, 1823 & corruíra \\
\hline Cistothorus platensis (Latham, 1790) & corruíra-do-campo \\
\hline Cantorchilus leucotis (Lafresnaye, 1845) & garrinchão-de-barriga-vermelha \\
\hline Cantorchilus longirostris (Vieillot, 1819) & garrinchão-de-bico-grande \\
\hline \multicolumn{2}{|l|}{ Donacobiidae Aleixo \& Pacheco, 2006} \\
\hline Donacobius atricapilla (Linnaeus, 1766) & japacanim \\
\hline \multicolumn{2}{|l|}{ Polioptilidae Baird, 1858} \\
\hline Ramphocaenus melanurus Vieillot, 1819 & bico-assovelado \\
\hline Polioptila lactea Sharpe, 1885 & balança-rabo-leitoso \\
\hline Polioptila dumicola (Vieillot, 1817) & balança-rabo-de-máscara \\
\hline \multicolumn{2}{|l|}{ Turdidae Rafinesque, 1815} \\
\hline Catharus fuscescens (Stephens, 1817) & sabiá-norte-americano \\
\hline Catharus ustulatus (Nuttall, 1840) & sabiá-de-óculos \\
\hline Turdus flavipes Vieillot, 1818 & sabiá-una \\
\hline Turdus rufiventris Vieillot, 1818 & sabiá-laranjeira \\
\hline Turdus leucomelas Vieillot, 1818 & sabiá-barranco \\
\hline Turdus amaurochalinus Cabanis, 1850 & sabiá-poca \\
\hline Turdus subalaris (Seebohm, 1887) & sabiá-ferreiro \\
\hline Turdus albicollis Vieillot, 1818 & sabiá-coleira \\
\hline \multicolumn{2}{|l|}{ Mimidae Bonaparte, 1853} \\
\hline Mimus saturninus (Lichtenstein, 1823) & sabiá-do-campo \\
\hline Mimus triurus (Vieillot, 1818) & calhandra-de-três-rabos \\
\hline
\end{tabular}


Tabela 1. Continuação...

\begin{tabular}{|c|c|}
\hline Nome do táxon & Nome em português \\
\hline \multicolumn{2}{|l|}{ Motacillidae Horsfield, 1821} \\
\hline Anthus lutescens Pucheran, 1855 & caminheiro-zumbidor \\
\hline Anthus correndera Vieillot, 1818 & caminheiro-de-espora \\
\hline Anthus nattereri Sclater, 1878 & caminheiro-grande \\
\hline Anthus hellmayri Hartert, 1909 & caminheiro-de-barriga-acanelada \\
\hline \multicolumn{2}{|l|}{ Coerebidae d'Orbigny \& Lafresnaye, 1838} \\
\hline Coereba flaveola (Linnaeus, 1758) & cambacica \\
\hline \multicolumn{2}{|l|}{ Thraupidae Cabanis, 1847} \\
\hline Saltator fuliginosus (Daudin, 1800) & pimentão \\
\hline Saltator maximus (Statius Muller, 1776) & tempera-viola \\
\hline Saltator similis d'Orbigny \& Lafresnaye, 1837 & trinca-ferro-verdadeiro \\
\hline Saltator maxillosus Cabanis, 1851 & bico-grosso \\
\hline Saltator aurantiirostris Vieillot, 1817 & bico-duro \\
\hline Saltatricula atricollis (Vieillot, 1817) & bico-de-pimenta \\
\hline Orchesticus abeillei (Lesson, 1839) & sanhaçu-pardo \\
\hline Schistochlamys melanopis (Latham, 1790) & sanhaçu-de-coleira \\
\hline Schistochlamys ruficapillus (Vieillot, 1817) & bico-de-veludo \\
\hline Cissopis leverianus (Gmelin, 1788) & tietinga \\
\hline Neothraupis fasciata (Lichtenstein, 1823) & cigarra-do-campo \\
\hline Nemosia pileata (Boddaert, 1783) & saíra-de-chapéu-preto \\
\hline Orthogonys chloricterus (Vieillot, 1819) & catirumbava \\
\hline Thlypopsis sordida (d'Orbigny \& Lafresnaye, 1837) & saí-canário \\
\hline Pyrrhocoma ruficeps (Strickland, 1844) & cabecinha-castanha \\
\hline Cypsnagra hirundinacea (Lesson, 1831) & bandoleta \\
\hline Trichothraupis melanops (Vieillot, 1818) & tiê-de-topete \\
\hline Eucometis penicillata (Spix, 1825) & pipira-da-taoca \\
\hline Tachyphonus cristatus (Linnaeus, 1766) & tiê-galo \\
\hline Tachyphonus coronatus (Vieillot, 1822) & tiê-preto \\
\hline Tachyphonus rufus (Boddaert, 1783) & pipira-preta \\
\hline Ramphocelus carbo (Pallas, 1764) & pipira-vermelha \\
\hline Ramphocelus bresilius (Linnaeus, 1766) & tiê-sangue \\
\hline Thraupis sayaca (Linnaeus, 1766) & sanhaçu-cinzento \\
\hline Thraupis cyanoptera (Vieillot, 1817) & sanhaçu-de-encontro-azul \\
\hline Thraupis ornata (Sparrman, 1789) & sanhaçu-de-encontro-amarelo \\
\hline Thraupis palmarum (Wied, 1823) & sanhaçu-do-coqueiro \\
\hline Thraupis bonariensis (Gmelin, 1789) & sanhaçu-papa-laranja \\
\hline Stephanophorus diadematus (Temminck, 1823) & sanhaçu-frade \\
\hline Pipraeidea melanonota (Vieillot, 1819) & saíra-viúva \\
\hline Tangara seledon (Statius Muller, 1776) & saíra-sete-cores \\
\hline Tangara cyanocephala (Statius Muller, 1776) & saíra-militar \\
\hline Tangara desmaresti (Vieillot, 1819) & saíra-lagarta \\
\hline Tangara cyanoventris (Vieillot, 1819) & saíra-douradinha \\
\hline Tangara cayana (Linnaeus, 1766) & saíra-amarela \\
\hline Tangara peruviana (Desmarest, 1806) & saíra-sapucaia \\
\hline Tangara preciosa (Cabanis, 1850) & saíra-preciosa \\
\hline Tersina viridis (Illiger, 1811) & saí-andorinha \\
\hline Dacnis nigripes Pelzeln, 1856 & saí-de-pernas-pretas \\
\hline Dacnis cayana (Linnaeus, 1766) & saí-azul \\
\hline Cyanerpes cyaneus (Linnaeus, 1766) & saíra-beija-flor \\
\hline Chlorophanes spiza (Linnaeus, 1758) & saí-verde \\
\hline Hemithraupis guira (Linnaeus, 1766) & saíra-de-papo-preto \\
\hline
\end{tabular}


Tabela 1. Continuação...

\begin{tabular}{|c|c|}
\hline Nome do táxon & Nome em português \\
\hline Hemithraupis ruficapilla (Vieillot, 1818) & saíra-ferrugem \\
\hline Conirostrum speciosum (Temminck, 1824) & figuinha-de-rabo-castanho \\
\hline Conirostrum bicolor (Vieillot, 1809) & figuinha-do-mangue \\
\hline \multicolumn{2}{|l|}{ Emberizidae Vigors, 1825} \\
\hline Zonotrichia capensis (Statius Muller, 1776) & tico-tico \\
\hline Ammodramus humeralis (Bosc, 1792) & tico-tico-do-campo \\
\hline Haplospiza unicolor Cabanis, 1851 & cigarra-bambu \\
\hline Donacospiza albifrons (Vieillot, 1817) & tico-tico-do-banhado \\
\hline Poospiza thoracica (Nordmann, 1835) & peito-pinhão \\
\hline Poospiza lateralis (Nordmann, 1835) & quete \\
\hline Poospiza cabanisi Bonaparte, 1850 & tico-tico-da-taquara \\
\hline Poospiza cinerea Bonaparte, 1850 & capacetinho-do-oco-do-pau \\
\hline Sicalis citrina Pelzeln, 1870 & canário-rasteiro \\
\hline Sicalis flaveola (Linnaeus, 1766) & canário-da-terra-verdadeiro \\
\hline Sicalis luteola (Sparrman, 1789) & tipio \\
\hline Emberizoides herbicola (Vieillot, 1817) & canário-do-campo \\
\hline Emberizoides ypiranganus Ihering \& Ihering, 1907 & canário-do-brejo \\
\hline Embernagra platensis (Gmelin, 1789) & sabiá-do-banhado \\
\hline Volatinia jacarina (Linnaeus, 1766) & tiziu \\
\hline Sporophila frontalis (Verreaux, 1869) & pixoxó \\
\hline Sporophila falcirostris (Temminck, 1820) & cigarra-verdadeira \\
\hline Sporophila plumbea (Wied, 1830) & patativa \\
\hline Sporophila collaris (Boddaert, 1783) & coleiro-do-brejo \\
\hline Sporophila lineola (Linnaeus, 1758) & bigodinho \\
\hline Sporophila nigricollis (Vieillot, 1823) & baiano \\
\hline Sporophila ardesiaca (Dubois, 1894) & papa-capim-de-costas-cinzas \\
\hline Sporophila caerulescens (Vieillot, 1823) & coleirinho \\
\hline Sporophila leucoptera (Vieillot, 1817) & chorão \\
\hline Sporophila bouvreuil (Statius Muller, 1776) & caboclinho \\
\hline Sporophila hypoxantha Cabanis, 1851 & caboclinho-de-barriga-vermelha \\
\hline Sporophila ruficollis Cabanis, 1851 & caboclinho-de-papo-escuro \\
\hline Sporophila palustris (Barrows, 1883) & caboclinho-de-papo-branco \\
\hline Sporophila cinnamomea (Lafresnaye, 1839) & caboclinho-de-chapéu-cinzento \\
\hline Sporophila melanogaster (Pelzeln, 1870) & caboclinho-de-barriga-preta \\
\hline Sporophila angolensis (Linnaeus, 1766) & curió \\
\hline Sporophila maximiliani (Cabanis, 1851) & bicudo \\
\hline Tiaris fuliginosus (Wied, 1830) & cigarra-do-coqueiro \\
\hline Arremon semitorquatus Swainson, 1838 & tico-tico-do-mato \\
\hline Arremon flavirostris Swainson, 1838 & tico-tico-de-bico-amarelo \\
\hline Charitospiza еисоsma Oberholser, 1905 & mineirinho \\
\hline Coryphaspiza melanotis (Temminck, 1822) & tico-tico-de-máscara-negra \\
\hline Coryphospingus cucullatus (Statius Muller, 1776) & tico-tico-rei \\
\hline Paroaria coronata (Miller, 1776) & cardeal \\
\hline Paroaria capitata (d'Orbigny \& Lafresnaye, 1837) & cavalaria \\
\hline \multicolumn{2}{|l|}{ Cardinalidae Ridgway, 1901} \\
\hline Piranga flava (Vieillot, 1822) & sanhaçu-de-fogo \\
\hline Habia rubica (Vieillot, 1817) & tiê-do-mato-grosso \\
\hline Cyanoloxia moesta (Hartlaub, 1853) & negrinho-do-mato \\
\hline Cyanoloxia brissonii (Lichtenstein, 1823) & azulão \\
\hline Cyanoloxia glaucocaerulea (d’Orbigny \& Lafresnaye, 1837) & azulinho \\
\hline $\begin{array}{l}\text { Parulidae Wetmore, Friedmann, Lincoln, Miller, Peters, van Rossem, Van Tyne } \\
\text { \& Zimmer } 1947\end{array}$ & \\
\hline
\end{tabular}




\begin{tabular}{|c|c|}
\hline Nome do táxon & Nome em português \\
\hline Parula pitiayumi (Vieillot, 1817) & mariquita \\
\hline Dendroica striata (Forster, 1772) & mariquita-de-perna-clara \\
\hline Geothlypis aequinoctialis (Gmelin, 1789) & pia-cobra \\
\hline Basileuterus culicivorus (Deppe, 1830) & pula-pula \\
\hline Basileuterus hypoleucus Bonaparte, 1830 & pula-pula-de-barriga-branca \\
\hline Basileuterus flaveolus (Baird, 1865) & canário-do-mato \\
\hline Basileuterus leucoblepharus (Vieillot, 1817) & pula-pula-assobiador \\
\hline Basileuterus leucophrys Pelzeln, 1868 & pula-pula-de-sobrancelha \\
\hline Phaeothlypis rivularis (Wied, 1821) & pula-pula-ribeirinho \\
\hline \multicolumn{2}{|l|}{ Icteridae Vigors, 1825} \\
\hline Psarocolius decumanus (Pallas, 1769) & japu \\
\hline Procacicus solitarius (Vieillot, 1816) & iraúna-de-bico-branco \\
\hline Cacicus chrysopterus (Vigors, 1825) & tecelão \\
\hline Cacicus haemorrhous (Linnaeus, 1766) & guaxe \\
\hline Icterus cayanensis (Linnaeus, 1766) & encontro \\
\hline Icterus croconotus (Wagler, 1829) & joão-pinto \\
\hline Gnorimopsar chopi (Vieillot, 1819) & graúna \\
\hline Amblyramphus holosericeus (Scopoli, 1786) & cardeal-do-banhado \\
\hline Agelasticus cyanopus (Vieillot, 1819) & carretão \\
\hline Chrysomus ruficapillus (Vieillot, 1819) & garibaldi \\
\hline Pseudoleistes guirahuro (Vieillot, 1819) & chopim-do-brejo \\
\hline Molothrus rufoaxillaris Cassin, 1866 & vira-bosta-picumã \\
\hline Molothrus oryzivorus (Gmelin, 1788) & iraúna-grande \\
\hline Molothrus bonariensis (Gmelin, 1789) & vira-bosta \\
\hline Sturnella superciliaris (Bonaparte, 1850) & polícia-inglesa-do-sul \\
\hline \multicolumn{2}{|l|}{ Fringillidae Leach, 1820} \\
\hline Sporagra magellanica (Vieillot, 1805) & pintassilgo \\
\hline Euphonia chlorotica (Linnaeus, 1766) & fim-fim \\
\hline Euphonia violacea (Linnaeus, 1758) & gaturamo-verdadeiro \\
\hline Euphonia chalybea (Mikan, 1825) & cais-cais \\
\hline Euphonia cyanocephala (Vieillot, 1818) & gaturamo-rei \\
\hline Euphonia pectoralis (Latham, 1801) & ferro-velho \\
\hline Chlorophonia cyanea (Thunberg, 1822) & bandeirinha \\
\hline \multicolumn{2}{|l|}{ Estrildidae Bonaparte, 1850} \\
\hline Estrilda astrild (Linnaeus, 1758) & bico-de-lacre \\
\hline \multicolumn{2}{|l|}{ Passeridae Rafinesque, 1815} \\
\hline Passer domesticus (Linnaeus, 1758) & pardal \\
\hline
\end{tabular}

(USP, UNICAMP e UNESP), além da Universidade Federal de São Carlos, UFSCar. Outras instituições que desenvolvem pesquisas na área são o Museu de Zoologia da USP, o Instituto Adolpho Lutz, a Fundação Florestal, o Instituto Florestal e o Museu de História Natural de Taubaté.

\section{Principais Acervos}

As instituições paulistas que abrigam espécimes e/ou amostras de tecido coletados no estado de São Paulo são: Museu de Zoologia da Universidade de São Paulo, São Paulo; o Museu de História Natural de Taubaté, Taubaté; o Museu de Zoologia da Universidade Estadual de Campinas "Adão José Cardoso", Campinas; a Coleção de Aves do Instituto Adolpho Lutz, Secretaria Estadual de Saúde, São Paulo; a Coleção Rolf Grantsau, São Bernardo do Campo; o Departamento de Genética e Biologia Evolutiva do Instituto de
Biociências da USP, São Paulo; e a Coleção de aves do Museu de História Natural, Universidade Estadual Paulista, Campus de Botucatu. Além disso, é importante ressaltar que muitas espécies também contam com registros documentados através das suas mais diferentes manifestações sonoras, depositadas principalmente no Arquivo Sonoro Neotropical (UNICAMP) e no Arquivo Sonoro do Museu de Zoologia da Universidade de São Paulo.

\section{Principais Lacunas do Conhecimento e Perspectivas de Pesquisa em Ornitologia para os Próximos 10 Anos}

A avifauna do estado de São Paulo pode ser considerada como bastante bem conhecida, com poucos registros novos para as aves terrestres do Estado previstos para os próximos anos. Novos registros para o estado devem se referir as espécies vagantes ou marinhas, que 
esporadicamente aparecem no litoral (e.g. Anous stolidus - Linnaeus, 1758).

Além disso, boa parte dos municípios paulistas possui algum inventário da sua avifauna, embora os resultados e as metodologias difiram muito entre si. As regiões oeste, norte e sudeste do Estado ainda apresentam lacunas de conhecimento importantes, e devem ser objetos de inventários mais intensos. Recomenda-se que esses inventários sejam padronizados e de médio-longo prazo (um ano ou mais), pois são esses estudos que vão fornecer dados essenciais para o manejo da paisagem e para a conservação, através do monitoramento das espécies ameaçadas de extinção.

São Paulo é o estado brasileiro com o maior número de espécies de aves ameaçadas de extincão (171, além de uma extinta, o patomergulhão, Mergus octosetaceus - Vieillot, 1817). Além disso, um número importante de espécies (47) foi considerado na categoria de "Quase ameaçadas", um dado preocupante, pois essas espécies podem ser as próximas a serem incluídas na lista de ameçadas de extincão (Silveira et al., 2009). Esses números são alarmantes e apontam para o desenvolvimento de pesquisas e restauração dos habitats nativos para tentar reverter esse quadro. Espécies que ocorrem no Cerrado (especialmente nos campos limpos) estão em situação gravíssima, e algumas delas (e.g. Columbina cyanopis Pelzeln, 1870, Eleothreptus candicans - Pelzeln, 1867 e Sporophila maximiliani - Cabanis, 1851) já podem ter seguido o mesmo caminho do pato-mergulhão. A ameaça às espécies se torna ainda mais grave quando consideramos o tempo de latência que muitos grupos podem apresentar. Há estudos que mostram que muitas espécies demoram várias décadas para responder aos distúrbios, como a perda e fragmentação do habitat (Uezu 2007, Metzger et al. 2009). Dessa forma, muitas espécies podem estar presentes no estado por uma questão de tempo, ou seja, serão extintas quando o sistema se estabilizar.

O monitoramento da avifauna em projetos que envolvam a restauração e a conexão dos milhares de fragmentos florestais se constitui numa linha de pesquisa importante visto que as aves formam um grupo relativamente fácil de ser amostrado e alguns grupos respondem rapidamente às alterações no ambiente. Embora o estado de São Paulo concentre uma das maiores coberturas de Mata Atlântica do país (FUNDAÇÃO SOS MATA ATLÂNTICA \& INSTITUTO NACIONAL DE PESQUISAS ESPACIAIS - INPE 2009), com exceção da Mata Atlântica de altitude nas Serras do Mar e Paranapiacaba, a situação de conservação dos demais ambientes naturais no estado pode ser considerada preocupante (Ribeiro et al. 2009). Muitas das comunidades de aves no interior do Estado, tanto no Cerrado (especialmente nos campos limpos) como na Mata Atlântica já possuem suas comunidades muito afetadas pelos processos de supressão, fragmentação e alteração do hábitat (Uezu et al. 2005, Develey \& Metzger 2005, Uezu 2007, Martensen et al. 2008). A expansão das atividades humanas sobre esses últimos remanescentes merece ser monitorada em projetos de médio-longo prazo.

Outro aspecto relevante é o estudo das aves em ambientes florestais artificiais. Uma parcela ainda pouco conhecida da avifauna é capaz de sobreviver ou utilizar plantios comerciais de eucalipto ou pinus, especialmente aqueles mais antigos. Essas áreas podem ser eventualmente utilizadas como "corredores artificiais" para conectar fragmentos de floresta nativa, se constituindo em um tema interessante de pesquisa.

Estudos que contemplem a variação geográfica, incluindo a delimitação de unidades evolutivas mais precisas e de filogeografia são importantes não só para refinar o nosso conhecimento sobre a formação e a evolução da avifauna, mas também para subsidiar de forma correta programas de reintrodução de espécies em locais onde elas foram extintas e não haja mais possibilidade de uma recolonização natural. Muitas espécies de aves exercem papel fundamental em processos ecológicos importantes como a polinização e a dispersão de sementes, e, em muitos casos, apenas com a reintrodução esses processos podem ser restaurados. Na atual situação das áreas naturais do estado, projetos de reintrodução realizados com base nos critérios propostos pela União Internacional para a Conservação da Natureza (IUCN, em inglês) devem também fazer parte dos programas mais amplos de conservação.

\section{Agradecimentos}

Aos colaboradores, especialmente ao Centro de Estudos Ornitológicos (CEO), em nome de Luiz Fernando de Andrade Figueiredo, que vem compilando os registros da avifauna de São Paulo, ao Rafael G. Pimentel e à Érica Hasui que disponibiliazaram seus bancos de dados de ocorrências de aves no Estado juntamente com as referências bibliográficas. Ao Instituto de Biociências e ao Museu de Zoologia da Universidade de São Paulo (IBUSP e MZUSP, respectivamente), ao CNPq, Fapesp e Fundação O Boticário, pelo apoio às nossas pesquisas. Edson Endrigo, pela gentileza em fornecer informações sobre os registros paulistas de Buteo albonotatus e Thraupis bonariensis.

\section{Referências Bibliográficas}

BERNARDO, C.S.S., RUBIM, P., DONATTI, C., MARQUES, R.M., DENZIN, C., GOBBO, S., STEFFLER, C.E., MEIRELLES, F., ANZOLIN, R.B., BOVENDORP, R., BUENO, R. \& GALETTI, M. No prelo. The plight of a large game birds: status of jacutinga populations (Cracidae, Aburria jacutinga) in the Atlantic rain forest. Stud. Avian Biol.

Boscolo, D., CANDiA-GALLARDO, C., AWADE, M. \& METZGER, J.P. 2008. Importance of inter-habitat gaps and stepping-stones for Lesser Woodcreepers (Xiphorhynchus fuscus) in the Atlantic Forest, Brazil. Biotropica 40(3):273-276. http://dx.doi.org/10.1111/j.17447429.2008.00409.x

CENTRO DE ESTUDOS ORNITOLÓGICOS - CEO. 2009. Lista de aves do estado de São Paulo. Versão: 5/2/2011. www.ceo.org.br (último acesso em 22/02/2011).

COMITÊ BRASILEIRO DE REGISTROS ORNITOLÓGICOS - CBRO. 2009. Listas das aves do Brasil - $8^{\text {a }}$ edição. http://www.cbro.org.br/CBRO/ pdf/avesbrasil_ago2009.pdf (último acesso em 22/02/2011).

DEVELEY, P.F. \& METZGER, J.P.W. 2005. Emerging threats to birds in Brazilian Atlantic forests: the roles of forest loss and configuration in a severely fragmented ecosystem. In Emerging threats to tropical forests (C.A. Peres \& W. Laurance, eds.). University of Chicago Press, Chicago.

FUNDAÇÃO SOS MATA ATLÂNTICA \& INSTITUTO NACIONAL DE PESQUISAS ESPACIAIS - INPE 2009. Atlas dos remanescentes florestais da Mata Atlântica: período 2005-2008 - Relatório parcial. Fundação SOS Mata Atlântica; Instituto Nacional de Pesquisas Espaciais, São Paulo.

MARTENSEN, A.C., PIMENTEL, R.G. \& METZGER, J.P. 2008. Relative effects of fragment size and connectivity on bird community in the Atlantic Rain Forest: implications for conservation. Biol. Cons. 141:2184-2192.

METZGER, J.P., MARTENSEN, A.C., DIXO, M., BERNACCI, L.C., RIBEIRO, M.C., TEIXEIRA, A.M.G. \& PARDINI, R. 2009. Time-lag in biological responses to landscape changes in a highly dynamic Atlantic forest region. Biol. Cons. 142:1166-1177. http://dx.doi.org/10.1016/j. biocon.2009.01.033 
RIBEIRO, M.C., METZGER, J.M., MARTENSEN, A.C. \& HIROTA, M.M. 2009. The Brazilian Atlantic Forest: how much is left, and how is the remaining forest distributed? Implications for conservation. Biol. Cons. 142:1141-1153. http://dx.doi.org/10.1016/j.biocon.2009.02.021

SILVA, W.R., SILVEIRA, L.F., UEZU, A., ANTUNES, A.Z., SUGIEDA, A.M., HASUI, E., FIGUEIREDO, L.F. \& DEVELEY, P.F. 2008. Aves. In Diretrizes para a conservação e restauração da Biodiversidade no Estado de São Paulo (R.R. Rodrigues \& V.L.R. Bononi, eds.). Instituto de Botânica; Imprensa Oficial do Estado de São Paulo, São Paulo, p.77$81+$ mapa.

SILVEIRA, L.F., BENEDICTO, G.A., SCHUNCK, F. \& SUGIEDA, A.M. 2009. Aves. In Fauna ameaçada de extinção no Estado de São Paulo: vertebrados (Bressan, P.M., Kierulff, M.C.M. \& Sugieda, A.M, eds.). Fundação Parque Zoológico de São Paulo: Secretaria do Meio Ambiente, São Paulo.
SILVEIRA, L.F., OLMOS, F. 2007. Quantas espécies de aves existem no Brasil? Conceitos de espécie, conservação e o que falta descobrir. Ararajuba. Rev. Bras. Orn. 15:289-296.

STOPIGLIA, R., STRAKER, L.C. \& RAPOSO, M.A. 2009. Kinglet Calyptura Calyptura cristata (Vieillot, 1818): documented record for the state of São Paulo and taxonomic status of the name Pipra tyrannulus Wagler, 1830. Br. Ornithol. Club 129(3):185-188.

UEZU, A. 2007. Composição e estrutura da comunidade de aves na paisagem fragmentada do Pontal do Paranapanema. Tese de doutorado, Universidade de São Paulo, São Paulo.

UEZU, A., METZGER, J.P. \& VIELLIARD, J.M.E. 2005. Effects of structural and functional connectivity and patch size on the abundance of seven Atlantic Forest bird species. Biol. Cons. 123:507-519. http://dx.doi. org/10.1016/j.biocon.2005.01.001 


\section{Apêndices}

Apêndice 1. Espécies de aves retiradas da lista de aves de São Paulo por não apresentarem registros documentados (Segundo Silveira et al., 2009, modificado). Appendix 1. Bird species removed from the list of birds of São Paulo which does not present documented record (according to Silveira et al. 2009, modified).

\begin{tabular}{|c|c|c|}
\hline Espécie & Nome popular & Motivo da retirada da lista de aves de São Paulo \\
\hline Buteo melanoleucus & águia-chilena & $\begin{array}{c}\text { Não apresenta registros confiáveis, embora seja de ocorrência provável, } \\
\text { especialmente na região noroeste. }\end{array}$ \\
\hline Aramides ypecaha & saracuruçu & Não apresenta qualquer registro documentado no estado. \\
\hline Touit surdus & $\begin{array}{l}\text { apuim-de- } \\
\text { cauda-amarela }\end{array}$ & $\begin{array}{l}\text { Espécie de difícil identificação, podendo ser facilmente confundida com Touit } \\
\text { melanonotus, que possui diversos registros documentados em São Paulo. Sua } \\
\text { inclusão na lista de São Paulo aguarda dados de melhor qualidade. }\end{array}$ \\
\hline Amazona rhodocorytha & chauá & $\begin{array}{l}\text { O registro dessa espécie foi realizado no Estado do Rio de Janeiro } \\
\left(23^{\circ} 17^{\prime} 24.20^{\prime \prime} \mathrm{S} \text { e } 44^{\circ} 38^{\prime} 30.21^{\prime \prime} \mathrm{W} \text {; P. Martuscelli, com. pess. }\right) \text { e } \\
\text { erroneamente "transferido" para São Paulo por ser algo próximo da divisa } \\
(\sim 20 \mathrm{~km}) \text { entre os dois Estados. Não foi registrado em São Paulo. }\end{array}$ \\
\hline Chordeiles pusillus & bacurauzinho & $\begin{array}{l}\text { Registro sem documentação, embora haja a possibilidade de sua ocorrência na } \\
\text { região noroeste do estado. }\end{array}$ \\
\hline Discosura langsdorffi & rabo-de-espinho & $\begin{array}{c}\text { Rara espécie de beija-flor, cujo limite sul é o Estado do Rio de Janeiro. A } \\
\text { inclusão dessa espécie na lista de SP baseia-se em uma única observação, sem } \\
\text { documentação. Sua inclusão na lista de São Paulo aguarda dados de melhor } \\
\text { qualidade. }\end{array}$ \\
\hline Cercomacra brasiliana & chororó-cinzento & $\begin{array}{l}\text { O registro foi realizado no Rio de Janeiro, próximo à fronteira com São Paulo. } \\
\text { Nunca foi observada no Estado. }\end{array}$ \\
\hline Tolmomyias flaviventris & bico-chato-amarelo & $\begin{array}{l}\text { Limite sul da distribuição no Rio de Janeiro. Não são conhecidos registros } \\
\text { documentados para São Paulo. }\end{array}$ \\
\hline Calyptura cristata & tietê-de-coroa & $\begin{array}{l}\text { Eventualmente citado por observadores de aves como ocorrente na região de } \\
\text { Ubatuba, mas nunca contou com qualquer documentação do registro. Uma } \\
\text { das aves mais raras e enigmáticas do Neotrópico; registros documentados } \\
\text { e confiáveis só foram realizados no Estado do Rio de Janeiro. A inclusão } \\
\text { dessa espécie na lista de São Paulo aguarda dados de melhor qualidade. } \\
\text { Stopiglia et al. (2009) apresentam um suposto registro documentado dessa } \\
\text { espécie para o estado com base nas coleções feitas por Sellow \& Olfers, que } \\
\text { sabidamente não são confiáveis com relação às localidades de coleta presentes } \\
\text { nos espécimes (Silveira et al., dados não publicados.). Dessa forma, Calyptura } \\
\text { cristata continua sem um registro confirmado para São Paulo. }\end{array}$ \\
\hline Dendroica cerulea & & $\begin{array}{c}\text { O único registro brasileiro tem como base uma única observação realizada na } \\
\text { EB de Boracéia, São Paulo. Sua inclusão na lista de São Paulo aguarda melhor } \\
\text { documentação. }\end{array}$ \\
\hline
\end{tabular}

Apêndice 2. Literatura consultada.

Appendix 2. Literature reviewed.

ALEIXO, A. 1997a. Composition of mixed-species bird flocks and abundance of flocking species in a semideciduous forest of southeastern Brazil. Ararajuba 5(1):11-18.

ALEIXO, A. 1997b. Estrutura e organização de comunidade de aves em áreas de Mata Atlântica e explorada por corte seletivo. Dissertação de mestrado, Universidade Estadual de Campinas, Campinas.

ALEIXO, A. \& GALETTI, M. 1997. The conservation of the avifauna in a lowland Atlantic Forest in south-east Brazil. Bird Cons. Intl. 7:235-261.

ALEIXO, A. \& SILVA, W.R. 1996. Estudo da diversidade de espécies de aves do Estado de São Paulo. Biota Neotrop.: http://www.biota.org.br/info/historico/ workshop/revisoes/aves.pdf (último acesso em 05/07/2010).

ALEIXO, A. \& VIELLIARD, J.M.E. 1996. Composição e dinâmica da avifauna da mata de Santa Genebra, Campinas, São Paulo, Brasil. Rev. Bras. Zool. 12(3):493-511.

ALLEGRINI, M.F. 1997. Avifauna como possível indicador biológico dos estádios de regeneração da Mata Atlântica. Dissertação de mestrado, Universidade de São Paulo, São Paulo.

ALMEIDA, A.F. 1979. Influência do tipo de vegetação nas populações de aves em uma floresta implantada de Pinus spp, na região de Agudos, SP. IPEF 18: 59-77.

ALMEIDA, A.F. 1981. Avifauna de uma área desflorestada em Anhembi, Estado de São Paulo, Brasil. Tese de doutorado, Universidade de São Paulo, São Paulo.

ALMEIDA, A.F. 2007. Aves do município de São Paulo. In Fauna Silvestre: quem são e onde vivem os animais na metrópole paulistana (A.F. Almeida \& M. Kawall, eds.). Secretaria Municipal do Verde e Meio Ambiente, São Paulo.

ALMEIDA, A.F., CARVALHO, M.A.S. \& SUMMA, M.E.L. 2003. Levantamento da avifauna da Região Metropolitana de São Paulo atendida pela Divisão Técnica de Medicina Veterinária e Manejo da Fauna Silvestre/DEPAVE/PMS. Bol. CEO 15:16-26.

ALMEIDA, M.E.C. 2002. Estrutura de comunidades de aves em áreas de cerrado da região nordeste do Estado de São Paulo. Tese de doutorado, Universidade Federal de São Carlos, São Carlos. 
ALVARENGA, H.M.F. 1990. Novos registros e expansões geográficas de aves no leste do estado de São Paulo. Ararajuba 1:115-117.

ANCIÃES, M. \& MARINI, M.A. 2000. The effects of fragmentation on fluctuating asymmetry in passerine birds of Brazilian tropical forests. J. Appl. Ecol. 37:1013-1028.

ANTUNES, A.Z. 2005. Alterações na composição da comunidade de aves ao longo do tempo em um fragmento florestal no sudeste do Brasil. Ararajuba 13(1):47-61.

ANTUNES, A.Z. \& WILLIS, E.O. 2003. Novos registros de aves para a fazenda Barreiro Rico, Anhembi-São Paulo. Ararajuba. 11:101-102.

AVANZO, V. \& SANFILIPPO, L.F. 2000. Levantamento preliminar da avifauna de Ilha Comprida, São Paulo. Bol. CEO 14: 10-14.

BANKS-LEITE, C. 2009. Efeitos da fragmentação lato sensu do habitat sobre a comunidade de aves de sub-bosque do Planalto Atlântico de Ibiúna e definição de espécies representativas. Tese de doutorado, Universidade de São Paulo, São Paulo.

BARBIERI, E. \& MENDONÇA, J.T. 2005. Distribuition and abundance of Charadridae at Ilha Comprida, São Paulo State, Brazil. J. Coastal Res. 21:e1-e10.

BARBIERI, E. \& PINNA, F.V. 2005. Distribuição da Batuíra-de-coleira (Charadrius collaris) durante o período de 1999 a 2001 na praia da Ilha Comprida. Rev. Bras. Orn. 13:25-31.

BARBOSA, A.F. 1982. Estudos preliminares para a reintrodução de espécies de aves no Parque Estadual de Campos do Jordão. Silv. São Paulo 16(3):1745-1750. BARBOSA, A.F. 1988. Avifauna do Parque Estadual de Campos do Jordão - São Paulo. Bol. Técn. IF. São Paulo 42:33-56.

BARBOSA, A.F. 1992. Avifauna de uma mata de Araucaria e Podocarpus do parque Estadual de Campos do Jordão, São Paulo. Dissertação de mestrado, Escola Superior de Agricultura Luiz de Queiroz, Piracicaba.

BARBOSA, A.F., MOLINA, M.J.C. \& TOLEDO, M.C.B. 1988. Análise preliminar da avifauna do Parque Estadual da Ilha do Cardoso - São Paulo. Bol. Técn. IF. São Paulo 42:181-203.

BENCKE, G.A., MAURICIO, G.N., DEVELEY, P.F. \& GOERCK, J.M. 2006. Áreas importantes para a conservação das aves no Brasil. Parte I - Estados do Domínio da Mata Atlântica. Save Brasil, São Paulo.

BETINI, G.S. 2001. Amostragem de aves por pontos numa floresta estacional semidecidual, São Paulo, Brasil. Dissertação de mestrado, Escola Superior de Agricultura Luiz de Queiroz, Piracicaba.

BEYER, D.D. 1999. Levantamento da avifauna de duas trilhas do Parque Estadual da Cantareira. Monografia de Bacharelado, Universidade Mackenzie, São Paulo.

BEYER, D.D. 2006. O papel dos bosques agroflorestais para a diversidade da avifauna na paisagem fragmentada do Pontal do Paranapanema, SP - Brasil. São Paulo, Dissertação de mestrado, Universidade de São Paulo, São Paulo.

BRUNO, L.A. 2010. Avifauna das florestas de restinga de Itanhaém/Mongaguá, Estado de São Paulo, Brasil. Atual. Ornitol. 153:50-54.

BUZATO, S. 1995. Estudo comparativo de flores polinizadas por beija-flores em três comunidades da Mata Atlântica no sudeste do Brasil. Tese de doutorado, Universidade Estadual de Campinas, Campinas.

BUZZETTI, D.R.C. 1996. Aves de floresta de restinga em Itanhaém, litoral sul do estado de São Paulo. Resumos V Congresso Brasileiro de Ornitologia, p. 17.

CAMARGO, H.F.A. 1946. Sobre uma pequena coleção de aves de Boracéia e do Varjão do Guaratuba. Pap. Avulsos Zool. 7(11):143-164.

CANDIDO-JUNIOR, J.F. 1991. Efeito da borda da mata sobre a composição da avifauna em Mata Residual em Rio Claro - SP. Dissertação de mestrado, Universidade Estadual Paulista, Rio Claro.

CARBONARI, M.P. 1990. Estudo da avifauna de um mosaico de vegetação natural e artificial no município de Rio Claro - SP. Dissertação de mestrado, Universidade Estadual Paulista Júlio de Mesquita Filho, Rio Claro.

CARVALHO, C.T. \& CARVALHO, J. 1992. A nidificação de Pionus maximiliani (Kuhl) e Ara maracana (Vieillot) em Gália São Paulo, Brasil (Aves, Psittacidae). Rev. Bras. Zool. 9(3-4):363-365. http://dx.doi.org/10.1590/S0101-81751992000200024

CASTRO, S.L.R. 1998. Ecologia do Tié-Sangue, Ramphocelus bresilius (Aves: Emberezidae: Thraupinae), no Parque Estadual da Serra do Mar, Núcleo Picinguaba, Ubatuba, SP. Dissertação de mestrado, Universidade Estadual Paulista Júlio de Mesquita Filho, Rio Claro.

CAVALHEIRO, M.L. 1999. Qualidade do ambiente e características fisiológicas do papagaio-de-cara-roxa (Amazona brasiliensis) Ilha Comprida - SP. Dissertação de mestrado, Universidade Federal do Paraná, Curitiba.

CAVARZERE, V., MORAES, G.P. \& DONATELLI, R.J. 2009. Diversidade de aves em uma mata estacional da região centro-oeste de São Paulo, Brasil. Rev. Bras. Bioc. 7(4):364-367.

CAZETTA, E., RUBIM, P., LUNARDI, V.O., FRANCISCO, M.R. \& GALETTI, M. 2002. Frugivoria e dispersão de sementes de Taluama ovata (Magnoliaceae) no sudeste brasileiro. Ararajuba 10(2):199-206.

CESTARI, C. 2001. Análise da dinâmica e composição da avifauna utilizando a técnica de redes ornitológicas de captura em duas áreas de um fragmento de mata estacional semidecídua presente no município de Lençóis Paulista - SP. Monografia, Universidade Estadual Paulista Júlio de Mesquita Filho, Bauru.

CESTARI, C. 2007. O uso de plantas epífitas por aves em uma região de Mata Atlântica no sudeste do Brasil. Dissertação de mestrado, Universidade Estadual Paulista Júlio de Mesquita Filho, Rio Claro.

CHRISTIANINI, A.V. 2001. Efeitos da estrutura de habitat sobre a predação de sementes por invertebrados, aves e mamíferos em uma Floresta Semidecídua de São Paulo. Dissertação de mestrado, Universidade Estadual Paulista Júlio de Mesquita Filho, Rio Claro.

CORTES, M.C. 2003. Frugivoria e dispersão de sementes de Euterpe edulis (Arecaceae) em três tipos florestais no Parque Estadual da Ilha do Cardoso - SP. Monografia, Universidade Estadual Paulista Júlio de Mesquita Filho, Rio Claro.

CORTES, M.C. 2006. Variação espacial nas interações entre o palmito Euterpe edulis e aves frugívoroas: implicações para a dispersão de sementes. Dissertação de mestrado, Universidade Estadual Paulista Júlio de Mesquita Filho, Rio Claro.

COSTA, J.C., BETINI, G.S. \& SILVA, W.S. 2004. Composição da comunidade de aves em uma área de cerradão e seu entorno na Estação ecológica de Assis, São Paulo. In III Simpósio Interno do Projeto Parcelas Permanentes. Ilha do Cardoso, São Paulo, p.123-131.

COSTA, T.V.V. 2002. Análise da variação anual na composição da avifauna na Fazenda Rio Claro, Lençóis Paulista, SP. Monografia, Universidade Estadual Paulista Júlio de Mesquita Filho, Bauru.

DÁRIO, F.R. 1999. Influência de corredor florestal entre fragmentos da Mata Atlântica utilizando-se a avifauna como indicador ecológico. Dissertação de mestrado, Escola Superior de Agricultura Luiz de Queiroz, Piracicaba.

DEVELEY, P.F. 1997. Ecologia de bandos mistos de aves de Mata Atlântica Estação Ecologica Juréia - Itatins. São Paulo, Brasil. Dissertação de mestrado, Universidade de São Paulo, São Paulo.

DEVELEY, P.F. 2004. Efeitos da fragmentação e do estado de conservação da floresta na diversidade de aves da mata Atlântica. Tese de doutorado, Universidade de São Paulo, São Paulo. 
DEVELEY, P.F. 2004. As aves da Estação Ecológica Juréia-Itatins. In Estação Ecológica Juréia-Itatins. Ambiente físico, flora e fauna (O.A.V. Marques \& W. Duleba, eds.). Holos, Ribeirão Preto.

DEVELEY, P.F \& ARGEL-DE-OLIVEIRA, M.M. 1996. Nova localidade para o gavião-asa-de-telha (Parabuteo Unicinctus) (Falconiformes: Accipteridae) no Estado de São Paulo, Brasil. Ararajuba 4:23-24.

DEVELEY, P.F. \& MARTENSEN, A.C. 2006. As aves da Reserva Florestal do Morro Grande (Cotia, SP). Biota Neotrop.: http://www.scielo.br/pdf/bn/v6n2/ v6n2a07.pdf (último acesso em 07/01/2010).

DEVELEY, P.F. \& PERES, C.A. 2000. Resource seasonality and the structure of mixed species bird flocks in a coastal Atlantic forest of southeastern Brazil. J. Trop. Ecol. 16:33-53.

DEVELEY, P.F., CAVANA, D.D. \& PIVELLO, V.G. 2005. Caracterização de Grupos Biológicos do Cerrado Pé-de-Gigante - Aves, p. 121-134. In O Cerrado Pé-de-Gigante: ecologia e conservação - Parque Estadual de Vassununga (V.R. Pivello \& E.M. Varanda, eds.). Secretaria do Meio Ambiente, São Paulo.

DIAS, M.M. 2000. Avifauna das Estações Ecológica de Jataí e Experimental de Luiz Antônio, São Paulo, Brasil. In Estação Ecológica de Jataí (J.E. Santos \& J.S.R. Pires, eds.). Rima, São Carlos, p.285-301.

DONATELLI, R.J., COSTA, T.V.V. \& FERREIRA, C.D. 2004. Dinâmica da avifauna em um fragmento de mata na Fazenda Rio Claro, Lençois Paulista, São Paulo, Brasil. Rev. Bras. Zool. 21(1): 97-114. http://dx.doi.org/10.1590/S0101-81752004000100017

DONATELLI, R.J., FERREIRA, C.D., DALBERTO, A.C., POSSO, S.R. 2007. Análise comparativa da assembléia de aves em dois remanescentes florestais no interior do Estado de São Paulo, Brasil. Rev. Bras. Zool. 24:362-375. http://dx.doi.org/10.1590/S0101-81752007000200015

DURIGAN, G., SIQUEIRA, M.F. \& FRANCO, G.A.D.C. 2007. Threats of the Cerrado remanescents of the State of São Paulo. Brazil. Sci. Agric. 64(4):355-363.

FADINI, R.F. 2005. Limitações bióticas afetando o recrutamento da palmeira Euterpe edulis em uma ilha continetal da Mata Atlântica. Dissertação de mestrado, Universidade Estadual Paulista Júlio de Mesquita Filho.

FERREIRA, M.A.P. 1994. Estudo comparado da dispersão e predação de sementes de Cabralea canjerana (Meliaceae) em duas áreas de mata do estado de São Paulo. Dissertação de mestrado, Universidade Estadual de Campinas, Campinas.

FIGUEIREDO, L.F.A. \& CUSTÓDIO-FILHO, A. 2003. Aves observadas em Unidades de Conservação do Estado de São Paulo por Antonio Flávio Barbosa. Bol. CEO 15:30-43.

FIGUEIREDO, L.F.A., GUSSONI, C.O.A. \& CAMPOS, R.P. 2000. Levantamento da avifauna do Parque Estadual Juquery, Franco da Rocha, São Paulo: uma avaliação auto-crítica das técnicas de campo para inventários ornitológicos. Bol. CEO 14:36-45.

FONSECA, R.C.B. 2005. Espécies-chave em um fragmento de floresta estacional semidecidual. Tese de doutorado, Universidade de São Paulo, São Paulo.

FRANCISCO, M.R. \& GALETTI, M. 2001. Frugivoria e dispersão de sementes de Rapanea lancifolia (Myrsinaceae) por aves numa área de cerrado do Estado de São Paulo, sudeste do Brasil. Ararajuba 9(1):13-19.

FRANCISCO, M.R. \& GALETTI, M. 2002a. Consumo dos frutos de Davilla rugosa (Dilleniaceae) por aves numa área de cerrado em São Carlos, Estado de São Paulo. Ararajuba 10(2):193-198.

FRANCISCO, M.R. \& GALETTI, M. 2002b. Aves como potenciais dispersoras de sementes de Ocotea pulchella Mart. (Lauraceae) numa área de vegetação de cerrado do sudeste brasileiro. Rev. Bras. Bot. 25(1):11-17. http://dx.doi.org/10.1590/S0100-84042002000100003

FRANCISCO, M.R., OLIVEIRA-JUNIOR, P.R.R. \& LUNARDI, V.O. 2008. Nest and fledglings of the Red-ruffed Fruitcrow (Pyroderus scutatus). The Wils. J. Ornithol. 120:413-416. http://dx.doi.org/10.1676/07-030.1

GABRIEL, V.A. 2005. Uso de cercas vivas por aves em uma paisagem fragmentada de Mata atlântica Semidecídua. Dissertação de mestrado, Universidade Estadual Paulista Júlio de Mesquita Filho, Rio Claro.

GABRIEL, V.A. \& PIZO, M.A. 2005. Foraging behavior of tyrant flycatchers (Aves, Tyrannidae) in Brazil. Rev. Bras. Zool. 22(4):1072-1077. http://dx.doi. org/10.1590/S0101-81752005000400036

GALETTI, M. \& ALEIXO, A.L.P. 1998. Effects of palm heart harvesting on avian frugivores in the Atlantic rain forest of Brazil. J. Appl. Ecol. 35:286-293.

GALETTI, M. \& PIZO, M.A. 1996. Fruit eating by birds in a forest fragment in southeastern Brazil. Ararajuba 4(2)71-79.

GALETTI, M., MARTUSCELLI, P., OLMOS, F. \& ALEIXO, A. 1997. Ecology and conservation of the Jacutinga Pipile jacutinga in the Atlantic Forest of Brazil. Biol. Cons. 82:31-39.

GALETTI, M., MARTUSCELLI, P., PIZZO, M.A. \& SIMÃO, I. 1997. Records of Harpy and Crested Eagles in the Brazilian Atlantic forest. Bull. Br. Ornithol. Club 117(1):27-31.

GALETTI, M., SCHUNCK, F., RIBEIRO, M., PAIVA, A.A., TOLEDO, R. \& FONSECA, L. 2006. Distribuição e Tamanho populacional do papagaio-de-cararoxa Amazona brasiliensis no estado de São Paulo. Rev. Bras. Orn. 14(3):239-247.

GOERCK, J.M. 1999a. Distribution of birds along an elevational gradient in the Atlantic forest of Brazil: implication for the conservation of endemic and endangered species. Bird Cons. Intl. 9:235-253.

GOERCK, J.M. 1999b. Ecology, evolution, and biogeography of Drymophila antbirds (Thamnophilidae, Aves) in the Neotropics. Tese de doutorado, University of Missouri, Columbia.

GOMES, V.S.M. 2001. Variação espaço-temporal de aves frugívoras no sub-bosque e chuva de sementes em um trecho de Mata Atlântica no Estado de São Paulo. Dissertação de mestrado, Universidade Estadual de Campinas, Campinas.

GONDIM, M.J.C. 1995. Dispersão de sementes de Trichilia sp (Meliaceae) por aves, em uma Mata Mesófila Semidecídua, no município de Rio Claro, SP Monografia, Universidade Estadual Paulista Júlio de Mesquita Filho, Rio Claro.

GONDIM, M.J.C. 2002. A exploração de frutos por aves frugívoras em uma área de cerradão no Estado de São Paulo. Tese de doutorado, Universidade Estadual Paulista Júlio de Mesquita Filho, Rio Claro.

GRAHAN, D.J. 1992. The avifauna of the Serra da Cantareira, São Paulo, Brazil: a preliminary survey. IF-Série Registros (10):1-56.

GRANZINOLLI, M.A.M., PEREIRA, R.G. \& MOTTA-JUNIOR, J.C. 2006. The Crowned Solitary-eagle Harpyhaliaetus coronatus (Accipitridae) in the cerrado of Estação Ecológica de Itirapina, southeast Brazil. Rev. Bras. Orn. 14(4):429-432.

GRANZINOLLI, M.A.M., KAMADA, B. \& BARROS, F.M. 2008. Monitoramento do gavião-asa-de-telha (Parabuteo unicinctus) na Baixada Santista, SP. In XVI Congresso Brasileiro de Ornitologia, Livro de Resumos, p.252, Palmas.

GUSSONI, C.O.A. \& CAMPOS, R.P. 2004. Avifauna da APA Federal da Bacia do Rio Paraiba do Sul nos municípios de Arujá e Santa Isabel. Atual. Ornitol. 117:11.

HASUI, E. 1994. O papel das aves frugívoras na dispersão de sementes em um fragmento de floresta estacional semidecídua secundária, em São Paulo, SP. Dissertação de mestrado, Universidade de São Paulo, São Paulo. 
HASUI, E. 2003. Influência da variação fisionômica da vegetação sobre a composição de aves frugívoras na Mata Atlântica. Tese de doutorado, Universidade Estadual de Campinas, Campinas.

HÖFLING, E. \& LENCIONI, F. 1992. Avifauna da Floresta Atlântica, Região de Salesópolis, Estado de São Paulo. Rev. Bras. Biol. 52(3):361-378.

IHERING, H. von. 1898a. As aves do Estado de São Paulo. Rev. Mus. Paul. 3:113-476.

IHERING, H. von. 1898b. Catalogue of the birds of São Paulo. Ibis 456-457.

ISHIKAWA-FERREIRA, L., RIBEIRO-NETO, F.B. \& HÖFLINGH, J.C. 1999. Avifauna aquática do reservatório de salto Grande e Varjão de Paulínia, Bacia do Rio Piracicaba, São Paulo, Brasil: espécies principais e variação temporal. Bioikos 13(1-2):7-18

KANEGAE, M.F., SILVA, M.T.M. \& DAMIANO, R.Z. 2008. Primeiro registro fotográfico do desenvolvimento de ninhegos de Tapaculo de Colarinho. Atual. Ornitol. 141:94-102.

KRISTOSCH, G.C. 1998. Biologia comportamental de Pyrrhura frontalis frontalis (Vieillot, 1817)-(Aves-Psittacidae): alimentação, interações sociais e reprodução. Dissertação de mestrado, Universidade Estadual Paulista Júlio de Mesquita Filho, Rio Claro.

LIMA, E.M.C. 2008. Taxonomia, distribuição e conservação dos “caboclinhos" do complexo Sporophila bouvreuil (Aves: Emberizidae). Dissertação de mestrado. Instituto de Biociências, Universidade de São Paulo.

LONGO, L.G.R. 2007. Análise da avifauna da RPPN Rio dos Pilões (Santa Isabel, SP), visando à conservação das espécies de um "Hotspot” da Mata Atlântica. Dissertação de mestrado, Escola Superior de Agricultura Luiz de Queiroz, Piracicaba.

LUNARDI, V.O. 2004. Análise genética molecular (RAPD) de Conopophaga melanops, Vieillot 1818 (Aves, Conopophagidae), em escala fina da Mata Atlântica e sua implicação para a conservação da espécie. Dissertação de mestrado, Universidade Federal de São Carlos, São Carlos.

MACHADO, C.G. 1999. A composição dos bandos mistos de aves na Mata Atlântica da Serra de Paranapiacaba, no sudeste brasileiro. Rev. Bras. Biol. 59(1):75-85.

MAGALHÃES, J.C.R. 1999. As aves na Fazenda Barreiro Rico. Editora Plêiade, São Paulo.

MARCELINO, V.R. 2002. Conservação ambiental e ações antrópicas em uma área de Ubatuba (SP). Dissertação de mestrado, Universidade de São Paulo, São Paulo.

MARCONDES-MACHADO, L.O. 2002. Comportamento alimentar de aves em Miconia rubiginosa (Melastomataceae) em fragmento de cerrado, São Paulo. Iheringia, Sér. Zool. 92(3):97-100. http://dx.doi.org/10.1590/S0073-47212002000300010

MARQUES, R.M. 2004. Diagnóstico das populações de aves e mamíferos cinegéticos do Parque Estadual da Serra do Mar, SP, Brasil. Dissertação de mestrado, Escola Superior de Agricultura Luiz de Queiroz, Piracicaba.

MARSDEN, S.J., WHIFFIN, M., SADGROVE, L. \& GUIMARAES-JUNIOR, P.R. 2003. Bird community composition and species abundance on two inshore islands in the Atlantic forest region of Brasil. Ararajuba 11(2):181-187.

MARTENSEN, A.C. 2008. Conservação de aves de sub-bosque em paisagens fragmentadas: importância da cobertura e da configuração do habitat. Dissertação de mestrado, Universidade de São Paulo, São Paulo.

MARTUSCELLI, P. 1992. Notas sobre aves pouco conhecidas do Estado de São Paulo. In Anais Encontro Nacional de Anilhadores de Aves 6:82-83.

MARTUSCELLI, P. 1995. Ecology and conservation of the Red-tailed Amazon Amazona brasiliensis in south-eastern Brazil. Bird Cons. Intl. 5:225-240.

MARTUSCELLI, P. 1996. Hunting behaviour of the Mantled Hawk Leucopternis polionota and the White-necked Hawk L. lacernulata in southeastern Brazil. Bull. Br. Ornithol. Club 116(2):114-116.

MARTUSCELLI, P. \& ANTONELLI, R. 1992. Novas adendas à avifauna do Estado de São Paulo. In Anais Encontro Nacional de Anilhadores Aves 6:82.

MARTUSCELI, P., OLMOS, F., GALETTI, M. 2001. Birds of Ilha do Cardoso State Park. Rio Claro, UNESP. http://www.rc.unesp.br/ib/ecologia/birdscardoso. html. (último acesso em 16/10/2001).

MATARAZZO-NEUBERGER, W.M. 1994. Guildas, organizacao e estrutura da comunidade: análise da avifauna da Represa Billings, São Paulo. Tese de doutorado, Universidade de São Paulo, São Paulo.

MINNS, J.C., BUZZETTI, D.R.C., ALBANO, C.G., WHITTAKER, A., GROSSET, A.E. \& PARRINI, R. 2010. Birds of Brazil/Aves do Brasil. DVD ROM. Editora Avis Brasilis, Vinhedo.

MOTTA-JUNIOR, J.C. 1990. Estrutura trófica e composição das avifaunas de três habitats terrestres na região central do estado de São Paulo. Ararajuba 1:65-71.

MOTTA-JUNIOR, J.C. \& GRANZINOLLI, M.A.M. 2004. Ocorrência de aves ameaçadas e endêmicas em fragmentos de cerrado no estado de São Paulo. In Cerrado (L.M. Coutinho \& W.B.C. Delitti). CD-ROM. Departamento de Ecologia - IBUSP.

MOTTA-JUNIOR, J.C. \& LOMBARDI, J.A. 1990. Aves como agentes dispersores da copaíba (Copaifera langsdorffi, Caesalpiniaceae) em São Carlos, estado de São Paulo. Ararajuba 1:105-106.

MOTTA-JUNIOR, J.C. \& VASCONCELLOS, L.A.S. 1996. Levantamento das aves do campus da Universidade Federal de São Carlos, estado de São Paulo, Brasil. In Anais do VII Seminário Regional de Ecologia. VII: 159-171. Programa de Pós-Graduação em Ecologia e Recursos Naturais.

MOTTA-JUNIOR, J.C., GRANZINOLLI, M.A.M. \& DEVELEY, P.F. 2008. Aves da estação ecológica de Itirapina, estado de São Paulo, Brasil. http://www. biotaneotropica.org.br/v8n3/pt/fullpaper?bn00308032008+pt (último acesso em: 08/07/2010).

MULLER, P. 1966. Studien zur Wierbeltirfauna der Insel von São Sebastião. Tese de doutorado. University of Saarbrucken, Saarbrucken.

NEVES, T., VOOREN, C.M. \& BASTOS, G. 2000. Proportions of Tristan and Wandering Albatrosses in incidental captures off the Brazilian coast. In Proceedings of the Second International Conference on the Biology and Conservation of Albatrosses and other Petrels, 8-12 May 2000, Honolulu, USA.

NUNES, M.F.C. 2000. Ecologia e conservação do maracanã-verdadeiro Propyrrhura maracana (Psittacidae) em um fragmento florestal no Sudeste do Brasil. Monografia, Universidade Estadual Paulista Júlio de Mesquita Filho, Rio Claro.

NUNES, M.F.C. \& GALETTI, M. 2007. Use of forest fragments by blue-winged macaws (Primolius maracana) within a fragmented landscape. Biodiv. Cons. 16:953-967. http://dx.doi.org/10.1007/s10531-006-9034-9

NUNES, M.F.C., GALETTI, M., MARSDEN, S., PEREIRA, R. \& PETERSON, A.T. 2007. Are large-scale distributional shifts of the blue-winged macaw (Primolius maracana) related to climate change? J. Biogeogr. 34:816-827.

OGRZEWALSKA, M.H. 2009. Efeito da fragmentação florestal na infestação por carrapatos (Acari: Ixodidae) em aves e infecção de carrapatos por Rickettsia spp no Pontal do Paranapanema, SP. Tese de doutorado, Universidade de São Paulo, São Paulo.

OLMOS, F. 1990. Frutificação de Chusquea meyeriana Rupr. (Poaceae, Bambusoideae) e dinâmica populacional de aves granívoras e roedores em área de Mata Atlântica. Dissertação de mestrado, Universidade Estadual de Campinas, Campinas.

OLMOS, F. 1996. Missing species in São Sebastião island, southeastern Brazil. Pap. Avulsos Zool. 39(18):329-349.

OLMOS, F. 2002. Non-breeding seabirds in Brazil: a review of band recoveries. Ararajuba 10:31-42. 
OLMOS, F. \& SILVA-E-SILVA, R. 2001. The avifauna of a southeastern Brazilian mangrove swamp. Intl. J. Orn. 4 (3):137-207.

OLMOS, F. \& SILVA-E-SILVA, R. 2003. Guará: ambiente, flora e fauna dos manguezais de Santos-Cubatão. Empresa das Artes, São Paulo.

OLMOS, F., MARTUSCELLI, P, SILVA-E-SILVA, R. \& NEVES, T.S. 1995. The sea birds of São Paulo, southeastern Brazil. Bull. Br. Ornithol. Club 115(2):117-128.

OLMOS, F., BASTOS, G.C.C. \& NEVES, T.S. 2000. Estimating seabird bycatch in Brazil. In Proceedings of the Second International Conference on the Biology and Conservation of Albatrosses and other Petrels, 8-12 May 2000, Honolulu, USA.

OLMOS, F., BUGONI, L., NEVES, T.S. \& PEPPES, F. 2006. Caracterização das aves oceânicas que interagem com a pesca de espinhel no Brasil. In Aves oceânicas e suas interações com a pesca na Região Sudeste-Sul do Brasil. (T. Neves, L. Bugoni, F. Olmos, C.M. Vooren \& C.L.B. Rossi-Wongtschowski, eds.). Universidade de São Paulo, São Paulo. (Série de documentos Revizee: Socre Sul / responsável Carmen Lúcia Del Bianco Rossi-Wongtschowski).

PACHECO, J.F. 1992. Levantamento da avifauna da Fazenda Bela Vista, Pontal, SP. R. 1. In II Congresso Brasileiro de Ornitologia. CBO. Resumos. Universidade Federal do Mato Grosso do Sul, Campo Grande.

PACHECO, J.F. 1994. O Interessante gavião-asa-de-telha (Parabuteo unicinctus) no Brasil. Um gavião raro? Atual. Ornitol. 6:13.

PACHECO, J.F. \& FONSECA, P.S.M. 2002. Resultados de excursão ornitológica a determinadas áreas dos estados de São Paulo, Santa Catarina e Rio Grande do Sul em janeiro, 1990. Atual. Ornitol. 106:3-5.

PACÍFICO, E. 2006. Registro do Guará-vermelho Eudocimus ruber (Ciconiiformes: Threskiornitidae), nos manguezais do rio Itanhaém, litoral centro-sul do Estado de São Paulo. In XIV Congresso Brasileiro de Ornitologia, Ouro Preto, MG, p. 15.

PALUDO, D., MARTUSCELLI, P., CAMPOS, F.P. 2004. Ocorrência de colônia reprodutiva de guará-vermelho Eudocimus ruber em Ilha Comprida no litoral do estado de São Paulo, Brasil. In XII Congresso Brasileiro de Ornitologia, Blumenau, SC, p. 323.

PARKER III, T.A. \& WILLIS, E.O. 1997. Notes on three tiny grassland flycatchers, with comments on the disappearance of South American fire-diversified savannas. Orn. Monogr. 48:549-555.

PASCOTTO, M.C. 2006. Avifauna dispersora de sementes de Alchornea glandulosa (Euphorbiaceae) em uma área de mata ciliar no estado de São Paulo. Rev. Bras. Orn. 14(3):291-296.

PASCOTTO, M.C. 2007. Rapaenea ferruginea (Ruiz \& Pav.) Mez. (Myrsinacceae) como uma importante fonte alimentar para as aves em uma mata de galeria no interior do Estado de São Paulo. Rev. Bras. Zool. 24(3):735-741.

PEDROCCHI, V., SILVA, C.R. \& SILVA, A. 2002. Check list of birds and mammals in the Paranapiacaba forest fragment. In Censuses of Vertebrates in a Brazilian Atlantic Rainforest Area: the Paranapiacaba Fragment (E. Mateos, J.C. Guix, A. Serra \& K. Pisciotta, eds.). Universitat de Barcelona, Barcelona.

PENSE, M.R. \& CARVALHO, A.P.C. 2005. Biodiversidade de aves do Parque Estadual do Jaraguá (SP). ConScientiae Saúde 4:55-62.

PENTEADO, M. 2006. Distribuição e abundância de aves em relação ao uso da terra na bacia do Rio Passa-Cinco, Estado de São Paulo, Brasil. Tese de doutorado, Escola Superior de Agricultura Luiz de Queiroz, Piracicaba.

PINTO, O.M.O. 1938. Catálogo das aves do Brasil e lista dos exemplares que as representam no Museu Paulista. 1a Parte. Secretaria da Agricultura, São Paulo. PINTO, O.M.O. 1944. Catálogo das aves do Brasil e lista dos exemplares existentes na coleção do Departamento de Zoologia. 2a Parte. Secretaria da Agricultura, São Paulo.

PINTO, O.M.O. 1949. Esboço monográfico dos Columbidae brasileiros. Arquivos de Zoologia do Estado de São Paulo, São Paulo.

PIZO, M.A. 1994. O uso de bromélias por aves na mata atlântica da Fazenda Intervales, sudeste do Brasil. Bromélia 1(4):3-7.

PIZO, M.A. 2003. Observations on a nest of Russet-winged Spadebill Platyrinchus leucoryphus in the Brazilian Atlantic forest. Cotinga 20:57-58.

PIZO, M.A. \& VIEIRA, E.M. 2004. Granivorous birds as potentially important post-dispersal seed predators in a brazilian forest fragment. Biotropica 36(3):417-423. http://dx.doi.org/10.1111/j.1744-7429.2004.tb00336.x

PIZO, M.A., SIMÃO, I. \& GALETTI, M. 1995. Diet and flock size of sympatric parrots in the Atlantic forest of Brazil. Orn. Neotrop. 6:87-95.

PIZO, M.A., SILVA, W.R., GALETTI, M. \& LAPS, R. 2002. Frugivory in cotingas of the Atlantic Forest of southeast Brazil. Ararajuba 10(2):177-185.

PONGILUPPI, T. \& SCHUNCK, F. 2007. Um caso de hibridização natural entre Amazona aestiva (LINNAEUS, 1766) e Amazona amazonica (LINNAEUS, 1758) na cidade de São Paulo, SP. In Resumos do XV Congresso Brasileiro de Ornitologia. Porto Alegre, RS.

POZZA, D.D. 2002. Composição da avifauna da Estação Ecológica de São Carlos (Brotas-SP) e reserva ambiental da Fazenda Santa Cecília (Patrocínio Paulista-SP). Dissertação de mestrado, Universidade Federal de São Carlos, São Carlos.

POZZA, D.D. 2002. Registros da avifauna ameaçada de extinção no nordeste do estado de São Paulo. Ararajuba 10(2):241-243.

RAGUSA-NETTO, J. 1999. Sócio-ecologia dos bandos mistos de aves em Campo Cerrado (Brotas, SP). Tese de doutorado, Universidade Estadual Paulista, Rio Claro.

REMOLD, H.G. \& RAMOS-NETO, M.B. 1995. A nest of restinga tyrannulet Phylloscartes kronei. Bull. Br. Ornithol. Club 115:239-240.

RODRIGUES, M. 1995. Spatial distribution and food utilization among tanagers in southeastern Brazil (Passeriforme: Emberezidae). Ararajuba 3:27-32.

ROSA, G.A.B. 2004. Frugivoria e dipersão de sementes por aves em uma área de reflorestamento misto em Botucatu, SP. Dissertação de mestrado, Universidade Estadual de Campinas, Campinas.

SANTOS, A.M.R. 2004. Comunidades de aves em remanescentes florestais secundários de uma área rural no sudeste do Brasil. Ararajuba 12(1):41-49.

SANTOS, A.M.R., FIGUEIREDO, L.F.A., PONTES, A. \& VISCONTI, M.A. 2000. Levantamento da avifauna da Estação Experimental de Itapetininga. Bol. CEO 14:63-66.

SÃO PAULO (Estado). 1998. Fauna ameaçada no Estado de São Paulo. Secretaria de Estado do Meio Ambiente. Série Documentos Ambientais.

SÃO PAULO (Município). 2010. Inventário da fauna do município de São Paulo 2010. Diário Oficial da cidade de São Paulo, ano 55 (94) - suplemento.

SCHAALMANN, C.T. 2004. Avaliação do conceito de habitat crítico por meio de levantamento de avifauna em duas áreas de cerrado no Estado de São Paulo. Dissertação de mestrado, Universidade de São Paulo, São Paulo.

SCHUNCK, F. 2005. Registro do trinta-réis-anão Sternula superciliaris, no reservatório Guarapiranga, localizado no município de São Paulo, SP. In XIII Congresso Brasileiro de Ornitologia. Belém, PA.

SCHUNCK, F. \& GHETTI, U. 2004. Reaparecimento do tuiuiú Jabiru mycteria (Ciconidae) na cidade de São Paulo após 110 anos sem registros. In XII Congresso Brasileiro de Ornitologia, Blumenau.

SILVA-E-SILVA, R. \& OLMOS, F. 1997. Parabuteo unicinctus (Falconiformes: Acciptridae) na Baixada Santista, litoral de São Paulo, Brasil. Ararajuba 5:76-79. 
SILVA, A.L. 1997. Relações entre padrões de forrageio, morfologia e uso de recursos no gênero Drymophila (Aves: Thamnophilidae). Dissertação de mestrado, Universidade de São Paulo, São Paulo.

SILVA, C.E.F., SANTIAGO, C.M., DESCIO, F. \& MARINO, L. 1990. Levantamento preliminar dos mamíferos, aves e répteis do Parque Estadual da Cantareira - SP. In VI Congresso Florestal Brasileiro. Campos do Jordão, 3:794-768.

SILVA, P.A. 2005. Predação de sementes pelo maracanã-nobre (Diopsittaca nobilis, Psittacidae) em uma planta exótica (Melia azedarach, Meliaceae) no oeste do Estado de São Paulo, Brasil. Rev. Bras. Orn. 13(2):183-185.

SILVA, W.R. 1992. As aves da Serra do Japi. In Historia natural da Serra do Japi: ecologia e preservação de uma área florestal no sudeste do Brasil (L.P.C. Morellato, ed.). Editora da UNICAMP, Campinas.

SILVA, W.R. 1988. Ornitocoria em Cereus peruvianus (Cactaceae) na Serra do Japi, Estado de São Paulo. Rev. Bras. Biol. 48(2):381-389.

SILVA, W.R. 2001. Padrões ecológicos, bioacústicos, biogeográficos e filogenético do complexo Basileuterus culicivorus (Aves, Parulidae) e demais espécies brasileiras do gênero. Tese de doutorado, Universidade Estadual de Campinas, Campinas.

SILVA, W.R. \& VIELLIARD, J. 2000. Avifauna de mata ciliar. In Matas ciliares: conservação e recuperação (R.R. Rodrigues \& H.F. Leitão-Filho, eds.). EDUSP; FAPESP, São Paulo, p.169-185.

SILVEIRA, L.F. 2009. As aves da Reserva Biológica do Alto da Serra de Paranapiacaba: uma revisão histórica do conhecimento ornitológico em uma reserva de Mata Atlântica do estado de São Paulo. In Reserva Biológica do Alto da Serra de Paranapiacaba (M.I.M.S. Lopes, M. Kirizawa \& M.M.R.F. Melo, eds.). Secretaria do Meio Ambiente, São Paulo, p.619-636.

SILVEIRA, L.F., OPPENHEIMER, M. \& SOBREIRA, C. 2006. Guia das aves da Fundação Maria Luisa e Oscar Americano. 1. ed. São Paulo: Fundação Maria Luisa e Oscar Americano. 1: 60.

SOUZA, F.L. 1995. Avifauna da cidade de Ribeirão Preto, Estado de São Paulo. Biotemas 8(2):100-109.

STRAUBE, F.C. \& SCHERER-NETO, P. 1995. Novas observações sobre o “cunhatai” Triclaria malachitacea (Spix, 1824) nos estados do Paraná e São Paulo (Psittacidae, Aves). Acta Biol. Leopold. 17(1):147-152.

STRAUBE, F.C., BORNSCHEIN, M.R. \& SCHERER-NETO, P. 1996. Coletânea da avifauna da região noroeste do estado do Paraná e áreas limítrofes (Brasil). Arq. Biol. Tecnol. 39(1):193-214.

TOLEDO, M.C.B. 1993. Avifauna em duas reservas fragmentadas de Mata Atlântica, na Serra da Mantiqueira-SP. Dissertação de mestrado, Escola Superior de Agricultura Luiz de Queiroz, Piracicaba.

UEZU A., BEYER D.D. \& METZGER, J.P.W. 2008. Can agroforest woodlots work as stepping stones for birds in the Atlantic Forest region? Biodiv. Cons. 17(8):1907-1922. http://dx.doi.org/10.1007/s10531-008-9329-0

UEZU, A. \& BETINI, G.S. 2003. Estudo da Avifauna do Parque Estadual do Morro do Diabo. Plano de manejo do Parque Estadual do Morro do Diabo. Governo do Estado de São Paulo; Secretaria do Meio Ambiente e Instituto Florestal, São Paulo.

VASCONCELOS, M.F. \& ROOS, A.L. 2000. Novos registros de aves para o Parque Estadual do Morro do Diabo, São Paulo. Melopsittacus 3(2):81-84.

VASCONCELOS, M.F. 2001. Estudo biogeográfico da avifauna campestre dos topos de montanha do sudeste do Brasil. Dissertação de mestrado, Universidade Federal de Minas Gerais, Belo Horizonte.

VIANNA, A.L.P. 2001. Análise da composição da avifauna associada às categorias alimentares na Estação Ecológica dos Caetetus, municípios de Gália e Alvilândia, São Paulo. Dissertação de mestrado, Universidade Estadual Paulista, Botucatu.

VIELLIARD, J.M.E. \& SILVA, W.R. 2001. Avifauna. In Intervales (C. Leonel, ed.). Fundação para a Conservação e a Produção Florestal do Estado de São Paulo, São Paulo.

WEGE, D.C. \& LONG, A.J. 1995. Key areas for threatened birds in the Neotropics. Birdl. Cons. Ser. 5:75-80.

WHITNEY, B.M., PACHECO, J.F. \& PARRINI, R. 1995. Two species of Neopelma in southeastern Brazil and diversification within the Neopelma/Tyranneutes complex: implications of the subspecies concept for conservation (Passeriformes: Tyrannidae). Ararajuba 3:43-53.

WHITNEY, B.M., PACHECO, J.F., ISLER, P.R. \& ISLER, M.L. 1995. Hylopezus natterei (Pinto, 1937) is a valid species (Passeriformes: Formicariidae). Ararajuba 3:37-42.

WILLIS, E.O. 1985. Behavior and systematic status of Gray-headed tanagers (Trichothraupis penicillata, Emberizidae). Naturalia 10:113-145.

WILLIS, E.O. 1979. The composition of avian communities in remanescent woodlots in Southern Brazil. Pap. Avulsos Zool. 33(1):1-25.

WILLIS, E.O. 1989. Mimicry in bird flocks of clould forests in southeas Brazil. Rev. Bras. Biol. 49(2):615-619.

WILLIS, E.O. 2003. Birds of a eucalyptus woodlot in interior São Paulo. Rev. Bras. Biol. 63(1):141-158. http://dx.doi.org/10.1590/S1519-69842003000100019

WILLIS, E.O. 2004. Birds of habitat spectrum in the Itirapina Savanna, São Paulo, Brazil (1982-2003). Braz. J. Biol. 64(4):901-910. http://dx.doi.org/10.1590/ S1519-6984200400050002

WILLIS, E.O. \& ONIKI, Y. 1981. Levantamento preliminar de aves em treze áreas do Estado de São Paulo. Rev. Bras. Biol. 41(1):121-135.

WILLIS, E.O., ONIKI Y. 1992. A new Phylloscartes (Tyrannidae) from southeastern Brazil. Bull. Br. Ornithol. Club 112(3):158-165.

WILLIS, E.O. \& ONIKI, Y. 1993. New and reconfirmed birds from the state of São Paulo, with notes on disappearing species. Bull. Br. Ornithol. Club 133(1):23-34.

WILLIS, E.O. \& ONIKI, Y. 2001. On a Nest of the Planalto Woodcreeper, Dendrocolaptes platyrostris, with Taxonomic and Conservation Notes. Wilson Bull. 113(2):231-233.

WILLIS, E.O. \& ONIKI, Y. 2002. Birds of a central São Paulo woodlot: 1. Censuses 1982-2000. Braz. J. Biol. 62(2):197-210. http://dx.doi.org/10.1590/ S1519-69842002000200003

WILLIS, E.O. \& ONIKI, Y. 2003. Aves do Estado de São Paulo. Editora Divisa, Rio Claro, SP, p.398.

ZACA, W. 2005. Composição da avifauna de um fragmento florestal de altitude no município de Atibaia (SP). Acta Biol. Leopold. 27(3):175-182. 